\title{
Kosterlitz-Thouless transition in helium films
}

\author{
G. Agnolet \\ Texas A\&M University, College Station, Texas 77843 \\ D. F. McQueeney* and J. D. Reppy \\ Laboratory of Atomic and Solid State Physics and the Materials Science Center, Cornell University, Ithaca, New York 14853
}

(Received 4 November 1988)

\begin{abstract}
The superfluid response of helium films with transition temperatures ranging from $70 \mathrm{mK}$ to 0.5 $\mathbf{K}$ has been studied using a torsional oscillator technique. A detailed analysis of the background indicates that the initial nonsuperfluid layer has an unexpected temperature-dependent period shift and dissipation. The superfluid response obeys a general scaling law which can be derived from the Kosterlitz-Thouless theory. Comparisons with the dynamic theory reveal nonuniversal behavior as a function of coverage.
\end{abstract}

\section{INTRODUCTION}

The superfluid phase transition in thin ${ }^{4} \mathrm{He}$ films on planar surfaces is generally acknowledged to be a realization of a Kosterlitz-Thouless ${ }^{1}$ (KT) two-dimensional (2D) phase transition. Unlike three-dimensional (3D) or bulk helium, the "ordered" or low-temperature phase does not exhibit true long-range order, but instead exhibits a power-law decay of the order-parameter correlation length. Even with only quasi-long-range order these films can still exhibit superfluidity as indicated by macroscopic persistent currents. In the Kosterlitz-Thouless theory, the disappearance of superfluidity is mediated by thermally excited vortices. At low temperatures, only a few vortices are present and these bind into vortexantivortex pairs which can be treated as a weakly interacting gas. As the density of vortex pairs increases with temperature, the interaction between members of those vortex pairs with large separations is screened by the presence of smaller vortex pairs. Eventually at some high temperature, the interaction becomes sufficiently weak that the large vortex pairs unbind. Once unbound vortices are present in the film, the correlation length exhibits only short-range order characteristic of a disordered state and the film no longer can support superfluid flow. Corresponding to this vortex unbinding transition, the superfluid areal density vanishes.

In order to interpret physical measurements, it is necessary to include the effects of a time-dependent flow velocity normally present in the experiments. Theoretical work by Ambegaokar, Halperin, Nelson, and Siggia (AHNS) (Ref. 2) and Huberman et al. ${ }^{3}$ have incorporated the effects of externally applied velocity fields on the dynamics of the vortex system. Their predictions have been confirmed in numerous experimental measurements employing a variety of techniques including torsional oscillators, ${ }^{4}$ quartz microbalance, ${ }^{5}$ third sound, ${ }^{6}$ and thermal transport. ${ }^{7,8}$

Most studies have concentrated on helium films with superfluid transition temperatures in excess of $1 \mathrm{~K}$ and with coverages greater than two atomic layers. In thermal transport measurements, it is the gas in contact with the film that conducts the heat through the system. This technique consequently becomes ineffective at low temperatures where the vapor pressure in equilibrium with the film is small. Third sound experiments can accurately measure the superfluid density, but it is not possible to follow the third-sound signal through the superfluid transition because of the large damping near $T_{c}$. In addition there are technical problems in coupling to third sound at very low temperatures and in very thin films. The advantage of the torsional oscillator technique is that it can measure the superfluid density from the lowest temperatures up through the superfluid transition with a sensitivity sufficient to examine helium films with superfluid coverages less than one monolayer.

In this paper we present torsional oscillator data taken on ${ }^{4} \mathrm{He}$ films with superfluid transition temperatures below $1 \mathrm{~K}$. In Sec. I, we review some of the theoretical ideas necessary for the analysis of our data and we introduce the concept of scaling which is clearly evident from the data. In Sec. II we describe the experimental apparatus and procedures. Section III contains our data and analysis. In the Summary we summarize our results and discuss their implications for future experiments. The model used to calculate coverages in this paper is discussed in detail in the Appendix.

\section{THEORETICAL BACKGROUND}

Our approach relies on physical arguments similar to the treatment by Young. ${ }^{9}$ For a more detailed discussion of the Kosterlitz-Thouless theory and its application to helium films, the reader is referred to a number of excellent review articles. ${ }^{10}$

\section{A. Static Kosterlitz-Thouless theory}

Although the vortex excitations in helium films are responsible for the disappearance of superfluidity, other thermal excitations such as phonons and rotons also influence the superfluid areal density. In particular, the initial decrease of the superfluid areal density from its 
$T=0$ value is solely due to these nonvortex excitations. It will be convenient for our discussions to define a background superfluid areal density $\rho_{s 0}(T)$ which includes all the effects of the nonvortex excitations. ${ }^{11}$ As we shall see $\rho_{s 0}(T)$ can be treated as a temperature-dependent coupling strength for the vortex interactions.

The vortex excitations consist of a quantized circulation flow field around a nonsuperfluid core. The size of this normal core is comparable to the coherence or healing length which defines the length scale over which the superfluid density can change from zero to its mean value. In bulk helium, the core radius is typically on the order of atomic dimensions $(\approx \AA)$ for temperatures far away from the superfluid transition. The spatial dependence of the flow field can be obtained from the quantization condition of the circulation. In order for the order parameter to be single valued, the flow must satisfy the condition

$$
\int_{\Gamma} \mathbf{v}_{\mathrm{s}} \cdot d \boldsymbol{l}=2 \pi n(\hbar / m)
$$

where $\Gamma$ is any closed contour and $n$ is an integer which specifies the amount of vorticity within the contour. Applying Eq. (1.1) to a single vortex, we find that the superfluid velocity around the vortex falls off as the inverse of the distance from the center; that is $v_{s}= \pm n(\hbar / m)(1 / r)$, where in this context $n$ can be thought of as the "charge" or strength of the vortex.

The energy of the vortex has two principal contributions; one comes from the kinetic energy of the flow field and the second comes from a temperature-dependent energy associated with the core $E_{c}(T)$,

$$
\begin{aligned}
E & =\frac{1}{2} \int_{r_{0}}^{R} d \mathbf{r} \rho_{s 0} v_{s}^{2}+E_{c}(T) \\
& =n^{2} \pi K_{0} \ln \left(R / r_{0}\right)+E_{c}(T),
\end{aligned}
$$

where $R$ is the size of the system and $K_{0}=\rho_{s 0}(T)(\hbar / m)^{2}$. In the above expression the core radius $r_{0}$ serves to cut off the divergence of the flow fields at small distances.

For macroscopic systems at low temperatures, a single isolated vortex would not be energetically favorable because its energy diverges with the size of the system. On the other hand, from entropy considerations one would expect high-temperature configurations to contain a large number of vortices. To estimate the crossover temperature for these two types of behavior, consider the change in the free energy due to the addition of one vortex to the film. Since the added vortex can be placed anywhere within the area of the system the change in entropy is $\Delta S=k_{B} \ln \left(R^{2} / r_{0}^{2}\right)$. Restricting our discussion to singly charged vortices $(n=1)$, the change in the free energy is given by $\Delta F=\Delta E-T \Delta S$ or $\Delta F \approx\left(\pi K_{0}\right.$ $\left.-2 k_{B} T\right) \ln \left(R / r_{0}\right)$. Therefore for $T<T^{*}=\pi K_{0} / 2 k_{B}$ the energy term dominates and free vortices are excluded from the system. Above $T^{*}$, the free energy for adding one vortex is negative and free vortices populate the system destroying correlations on long length scales.

To correctly treat the interactions of the vortex system one must include the Bernoulli or Magnus forces on a vortex induced by the flow field of the other vortices. For oppositely charged vortices, this interaction is attractive and provides the binding energy of the vortex pair. Since the combined velocity field of two oppositely charged vortices decays at large distances more rapidly than $(1 / r)$, the kinetic energy of the total flow field no longer diverges with the size of the system but instead remains finite. To first order, the energy of the bound pair depends logarithmically on the separation $r$ between the vortices,

$$
E_{\text {pair }}=2 \pi n^{2} K_{0} \ln \left(r / r_{0}\right)+2 E_{c}(T) .
$$

Given a finite self-energy, bound vortex pairs can exist in the film even at low temperatures. In the limit of a small number of pairs so that interactions between pairs can be ignored, the equilibrium density of pairs with a given separation $r$ can be described by a Boltzmann distribution. Assuming individual vortex cores do not overlap, the number of possible sites for the first vortex is just the area of the system divided by the area of the core: $R^{2} / r_{0}^{2}$. Similarly the number of possible sites for the second vortex of the pair within an annular region a distance $r$ from the first is $2 \pi r d r / r_{0}^{2}$. Using the expression for the energy of a pair, the areal density for vortex pairs with separation $r$ can therefore be approximated by

$$
\begin{aligned}
n(r) & =\left(2 \pi r / r_{0}^{4}\right) \exp \left(-E_{\text {pair }} / k_{B} T\right) \\
& =\left(2 \pi r / r_{0}^{4}\right) y_{0}^{2} \exp \left[-V(r) / k_{B} T\right],
\end{aligned}
$$

where $y_{0}=\exp \left[-E_{c}(T) / k_{B} T\right]$ serves as the fugacity of the vortices and $V(r)=2 \pi K_{0} \ln \left(r / r_{0}\right)$ is the interaction potential described previously.

As the density of vortex pairs becomes larger at higher temperatures, Eq. (1.4) must be modified to include the interactions between the pairs. The interaction between members of a pair with separation $r$ is screened by intermediate pairs with smaller separations $r^{\prime}<r$ situated between them. This is analogous to the reduction in the force between two oppositely charged particles placed within a dielectric medium. As the electric field of the exterior charges aligns the electric dipoles in the dielectric, the field from the aligned dipoles reduces the initial electric field and thereby decreases the interaction between the original charges. The same process occurs in the vortex system, only now it is the flow fields of the large pairs which polarize the smaller pairs.

Starting with the equilibrium Boltzmann distribution of pairs one can show that the polarizability of vortex pairs with separation $r$, is given by $\alpha(r)=\pi r^{2} K_{0} / 2 k_{B} T$ in the weak-field limit $\left(r \hbar \rho_{s 0} v_{s} / m<<k_{B} T\right)$. From this polarizability one defines a length-dependent dielectric function $\epsilon(r)$ which relates the renormalized force between vortices separated by a distance $r$ to its unscreened value [i.e., $-2 \pi K_{0} / r$ becomes $\left.-2 \pi K_{0} / r \epsilon(r)\right]$,

$$
\begin{aligned}
& \epsilon(r)=1+4 \pi \chi(r) \\
& \chi(r)=\int_{r_{0}}^{r} d \mathbf{r}^{\prime} \alpha\left(r^{\prime}\right) n\left(r^{\prime}\right),
\end{aligned}
$$

where $\chi(r)$ is the susceptibility of all pairs with separation less than $r$. Modifying our previous expression for $n(r)$ we obtain 


$$
n(r)=\left(2 \pi r / r_{0}^{4}\right) y_{0}^{2} \exp \left[-V^{\prime}(r) / k_{B} T\right],
$$

where

$$
V^{\prime}(r)=2 \pi K_{0} \int_{r_{0}}^{r} d \mathbf{r}^{\prime} / r^{\prime} \epsilon\left(r^{\prime}\right) .
$$

Equations (1.5) and (1.6) define a self-consistent means by which the effective dielectric constant can be evaluated in the thermodynamic limit $r \rightarrow \infty$.

For convenience, the following length-dependent parameters can be defined as

$$
\begin{aligned}
& K^{-1}(l)=\epsilon(r) k_{B} T K_{0}^{-1}, \\
& y(l)=y_{0}\left(r / r_{0}\right)^{2} \exp \left[-V^{\prime}(r) / 2 k_{B} T\right], \\
& l=\ln \left(r / r_{0}\right) .
\end{aligned}
$$

With these definitions Eqs. (1.5) and (1.6) can be rewritten in terms of $y(l)$ and $K(l)$,

$$
\begin{aligned}
& K^{-1}(l)=k_{B} T K_{0}^{-1}+4 \pi^{3} \int_{0}^{l} d l^{\prime} y^{2}\left(l^{\prime}\right), \\
& y(l)=y_{0} \exp \left[2 l-\pi \int_{0}^{l} d l^{\prime} K\left(l^{\prime}\right)\right],
\end{aligned}
$$

or, equivalently, in differential form,

$$
\begin{aligned}
& d K^{-1}(l) / d l=4 \pi^{3} y^{2}(l), \\
& d y(l) / d l=y(l)[2-\pi K(l)],
\end{aligned}
$$

with initial conditions $K(l)=K_{0} / k_{B} T$ and $y(l)=y_{0}$ at $l=0$. These equations form the recursion relations first applied to the problem of $2 \mathrm{D}$ superfluidity of Kosterlitz and Thouless.

$K(l)$ represents the interaction between vortices separated by a distance $r_{0} e^{l}$ once the screening effects from vortex pairs with separations less than $r_{0} e^{l}$ have been taken into account. This rescaling of the interaction also reflects a change in the superfluid density of the film. The quantity $K(l \rightarrow \infty)$ by definition incorporates the effects of vortex pairs on all length scales and can be shown to be proportional to the macroscopic superfluid density of the film. Explicitly one has that

$$
\begin{aligned}
& K^{-1}(l \rightarrow \infty)=k_{B} T K_{0}^{-1} \epsilon(l \rightarrow \infty), \\
& \rho_{s}(T)=\epsilon^{-1}(l \rightarrow \infty) \rho_{s 0}(T) .
\end{aligned}
$$

The quantity $y(l)$ is related to the areal density of vortex pairs with separations $r_{0} e^{l}$. Large values of $y(l)$ indicate a high density of vortex pairs, while either a small or zero value corresponds to only a few or no pairs.

Solving for $K^{-1}(l)$ in terms of $y(l)$ from Eq. (1.9) we obtain

$$
\frac{d K^{-1}(l)}{d y(l)}=\frac{4 \pi^{3} y(l)}{[2-\pi K(l)]},
$$

which can be integrated to obtain

$2 \pi^{2} y^{2}(l)-(2 / \pi) K^{-1}(l)+\ln \left[K^{-1}(l) / \pi\right]=C(T)$,

where $C(T)$ is the temperature-dependent integration constant determined by the initial conditions at $l=0$, i.e., $y(l \rightarrow 0)=y_{0}$ and $K^{-1}(l \rightarrow 0)=k_{B} T K_{0}^{-i}$,

$$
\begin{aligned}
C(T)= & 2 \pi^{2} y_{0}^{2}-(2 / \pi) k_{B} T K_{0}^{-1} \\
& +\ln \left(k_{B} T K_{0}^{-1} / \pi\right),
\end{aligned}
$$

or expressing $C(T)$ in terms of the core energy $E_{c}(T)$ and the background superfluid density $\rho_{s 0}(T)$,

$$
\begin{aligned}
C(T)= & 2 \pi^{2} \exp \left[-2 E_{c}(T) / k_{B} T\right]-2\left(\frac{k_{B} T}{\pi \rho_{s 0}(T)(\hbar / m)^{2}}\right) \\
& +\ln \left(\frac{k_{B} T}{\pi \rho_{s 0}(T)(\hbar / m)^{2}}\right) .
\end{aligned}
$$

By substituting for $y^{2}(l)$ in Eq. (1.9) from Eq. (1.12), we obtain our final equation for $K^{-1}(l)$,

$$
\frac{d K^{-1}(l)}{d l}=4 K^{-1}(l)-2 \pi \ln \left[K^{-1}(l) / \pi\right]+2 \pi C(T) .
$$

The family of trajectories in the $\left(K^{-1}, y\right)$ plane defined by Eq. (1.12) is shown in Fig. 1. Each trajectory corresponds to a different temperature with arrows indicating the direction of increasing $l$. The locus of initial conditions corresponding to $l=0$ at different temperatures is indicated by the dotted line.

Consider the trajectory marked $A$. As the value of $l$ increases, we integrate out vortex pairs over larger and larger length scales until for $l=\infty$ one reaches the $y=0$ axis corresponding to no vortex pairs with an infinite separation. Since the trajectory intersects the axis at some finite value of $K^{-1}, \rho_{s}$ remains nonzero although it is smaller than its initial value $\rho_{s 0}$. We interpret trajectories of this type to describe the ordered state with $T<T_{c}$. The behavior of trajectory $C$ is dramatically different. As $l$ increases, the trajectory initially flows towards smaller values of $y$ but eventually changes direction and flows towards increasing values of $y$ and $K^{-1}$. The large values

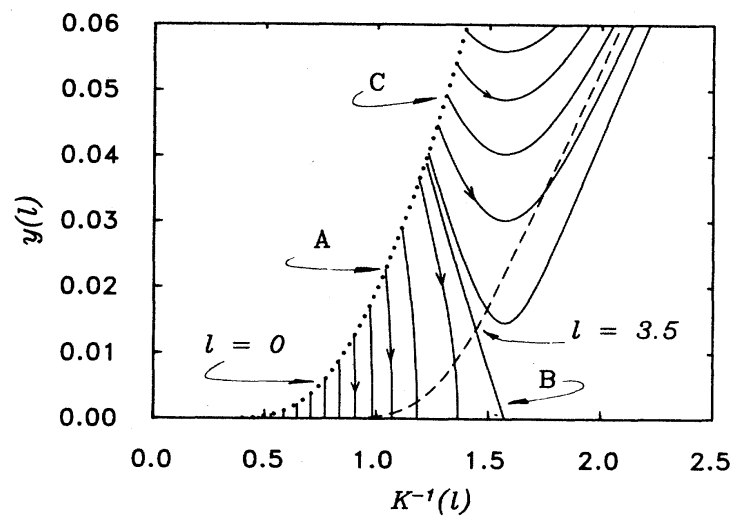

FIG. 1. Flow trajectories derived from the KosterlitzThouless recursion relations plotted in the $y(l), K^{-1}(l)$ plane. Trajectories marked $A, B$, and $C$ correspond to $T<T_{c}, T_{c}$, and $T>T_{c}$, respectively. The dotted line marked $l=0$ represents the initial microscopic values of $y(l)$ and $K^{-1}(l)$, whereas the dashed line represents their values at a dynamic length scale of $l_{D}=3.5$. 
of $y$ indicate a high density of vortices and suggests that the system is now in a disordered state. For this trajectory $K^{-1}$ diverges and the superfluid density vanishes. The single trajectory which separates these two regions of behavior is labeled $B$ and defines the transition temperature for this system. The temperature corresponding to this trajectory is the highest temperature for which there exists a finite intersection on the $y=0$ axis and therefore a nonzero $\rho_{s}$. Since any higher-temperature trajectories flow toward $\rho_{s} \rightarrow 0$ the superfluid density must have a discontinuity at $T_{c}$. Nelson and Kosterlitz ${ }^{12}$ have shown from an analysis of the recursion relations (1.9) that

$$
\lim _{T \rightarrow T_{c}} K^{-1}(l \rightarrow \infty) \rightarrow \pi / 2,
$$

defines a universal constant for this system,

$$
\lim _{T \rightarrow T_{c}}\left[\rho_{s}(T) / T\right] \rightarrow\left[\rho_{s}\left(T_{c}^{-}\right) / T_{c}\right]=\frac{2 m^{2} k_{B}}{\pi \hbar^{2}} .
$$

At the transition temperature only the vortex pairs with an infinite separation can unbind. Pairs with smaller separations do not unbind until much higher temperatures. An estimate of the temperature at which the vortex pairs of a given separation unbind can be made from the behavior of the $T>T_{c}$ trajectories in Fig. 1. The divergence of $y(l)$ implies a large number of vortices in the film. The length scale at which $y(l)$ becomes equal to its initial value at $l=0, y_{0}$, can be used to define a hightemperature correlation length $\xi_{+}$beyond which vortices are no longer correlated with other vortices and are considered to be unbound. Because $\xi$ + will be approximately equal to the distance between free vortices in the film the density of free vortices, $n_{\text {free }}$, can be estimated by

$$
n_{\text {free }}=F \xi_{+}^{-2}
$$

where the constant $F$ is introduced in the definition because of the arbitrariness in determining whether a vortex is bound or free. The value of $F$ is expected to be on the order of unity.

\section{B. Dynamic theory}

The Kosterlitz-Thouless theory as described in Sec. I A assumes that the system of interacting vortices is in thermal equilibrium and does not apply to systems subjected to externally applied flow fields. However, the superfluid density is inherently the dynamic response of the system to an external velocity field. Third sound involves both nonzero frequency and wave number excitations of the superfluid. Oscillating substrate experiments also have oscillating velocity fields (nonzero frequency). Perhaps closest to the static limit are thermally or hydrostatically driven flow and persistent current experiments although the latter do involve time-dependent flow fields. In order to make contact between theory and experiment one must include within the framework of the KT theory the effects of an external field on the motion of the vortices. In effect one must calculate the superfluid density $\rho_{s}(T, \omega, k)$ as a function of frequency and wavelength. In this section we shall describe the theory of
AHNS and its relation to the oscillator experiments.

In the static theory the polarizability was based on an equilibrium distribution of the vortex pairs. Now if instead of a steady flow, the velocity field oscillates with frequency $\omega$, then a vortex pair may not have sufficient time to completely reorient itself to the flow field before the flow changes direction. Whether this occurs for a particular pair will depend upon the dynamics of its motion within the film. For simplicity one can include these effects by multiplying the polarizability $\alpha(r)$ by a dynamic response function $g(r, \omega)$ which will in general depend upon the size of the vortex pair. With this ansatz, we can follow our previous formalism and define a frequency-dependent susceptibility and dielectric function,

$$
\begin{aligned}
& \chi(r, \omega)=\int_{r_{0}}^{r} d \mathbf{r}^{\prime} \alpha\left(r^{\prime}\right) n\left(r^{\prime}\right) g\left(r^{\prime}, \omega\right), \\
& \epsilon(r, \omega)=1+4 \pi \chi(r, \omega) .
\end{aligned}
$$

As before these equations, together with the equations for $n(r)$, can be solved to obtain the dielectric function of the system. Generalizing our definition of $\rho_{s}$ we obtain,

$$
\rho_{s}(T)=\rho_{s 0}(T) / \epsilon(\omega),
$$

where $\epsilon(\omega)=\lim _{r \rightarrow \infty} \epsilon(r, \omega)$ incorporates the dynamic response of vortices on all length scales. Since some vortices may not be able to respond instantaneously to changes in the flow direction, the phase lag between the velocity fields of the vortices and the external flow will in general make $\epsilon(\omega)$, and consequently $\rho_{s}(T)$, complex. We interpret the real part of $\rho_{s}$ to be that fraction of the film which moves in phase with the driving velocity field while the imaginary part of $\rho_{s}$ to be that fraction which moves $90^{\circ}$ out of phase.

To understand the significance of the real and imaginary parts of $\rho_{s}$ consider the physical situation of an oscillating substrate experiment. Viewed as a simple harmonic oscillator of mass $M$, spring constant $k$ and an internal dissipation $\gamma$, the substrate has an equation of motion $\left[-\omega^{2}(M+A \rho)+i \omega \gamma+k\right] X=0$ where we have assumed that the displacement of the oscillator $X$ is harmonic, i.e., $X(t)=X_{0} e^{i \omega t}$. $\rho A$ is the mass of adsorbed helium and $A$ is the area of the substrate. When the film becomes superfluid, $A \rho$ is replaced by $A\left(\rho-\rho_{s}\right)$ since the superfluid component will not move with the substrate. Writing $\rho_{s}$ in terms of its real and imaginary parts,

$$
\begin{aligned}
& \left(-\omega^{2}\left\{M+A \rho-A \rho_{s 0} \operatorname{Re}\left[\epsilon^{-1}(\omega)\right]\right\}\right. \\
& \left.\quad+i \omega\left\{\gamma+\omega A \rho_{s 0} \operatorname{Im}\left[\epsilon^{-1}(\omega)\right]\right\}+k\right)=0,
\end{aligned}
$$

where in the limit of small damping the resonant period of the oscillator is given by

$$
P^{2}=(2 \pi)^{2}\left\{M+A \rho-A \rho_{s 0} \operatorname{Re}\left[\epsilon^{-1}(\omega)\right]\right\} / k .
$$

Denoting $P_{0}$ to be the period of the oscillator with all of the helium moving with the substrate we can define the reduced period to be

$2 \Delta P / P=\left(P^{2}-P_{0}^{2}\right) / P_{0}^{2}=(A / M) \rho_{s 0} \operatorname{Re}\left[\epsilon^{-1}(\omega)\right]$. 
Clearly the imaginary part of $\rho_{s}$ adds to the internal dissipation of the oscillator and can be written in terms of the $Q$ or quality factor of the oscillator,

$$
\Delta Q^{-1}=Q^{-1}-Q_{0}^{-1}=(A / M) \rho_{s 0} \operatorname{Im}\left[\epsilon^{-1}(\omega)\right],
$$

where $Q_{0}=M \omega / \gamma$ is the $Q$ of the empty cell. (Note that in both expressions the approximation $M \gg A \rho_{s}$ has been made.)

To proceed further one must determine the explicit form of $g(r, \omega)$ from the equations of motion for vortices. In addition to the Magnus forces induced by external superfluid flow fields there exists a drag between the core of the vortex and the normal excitations of the film. Hall and Vinen ${ }^{13}$ give an explicit form for these drag forces,

$$
F_{\mathrm{drag}}=B\left(\mathbf{v}_{\mathbf{n}}-d \mathbf{r} / d t\right)+B^{\prime} n \mathbf{z} \times\left(\mathbf{v}_{\mathbf{n}}-d \mathbf{r} / d t\right),
$$

where $B$ and $B^{\prime}$ are phenomenological drag coefficients and $n$ is the charge of the vortex. To obtain an equation of motion AHNS assume that the vortices achieve a drift velocity determined by the balance between the Magnus force and the drag forces. The resulting average velocity of a vortex is therefore given by

$$
\begin{aligned}
d \mathbf{r} / d t= & n\left(D / k_{B} T\right)\left(2 \pi \hbar \rho_{s 0} / m\right)\left[\mathbf{z} \times\left(\mathbf{v}_{\mathbf{n}}-\mathbf{v}_{\mathbf{s}}\right)\right] \\
& +C\left(\mathbf{v}_{\mathbf{n}}-\mathbf{v}_{\mathbf{s}}\right)+\mathbf{v}_{\mathbf{s}},
\end{aligned}
$$

where both $D$ and $C$ can be expressed in terms of $B$ and $B^{\prime}$. Because the normal component in thin helium films can be assumed to be clamped to the substrate, $\mathbf{v}_{\mathbf{n}}$ will be taken to zero. The coefficient $D$ is interpreted as a diffusion constant and reflects the fact that on long time scales the vortices behave as diffusive particles. The second and third terms in Eq. (1.26) carry the vortices in the direction of $\mathbf{v}_{\mathbf{s}}$ and therefore do not directly contribute to changes in $\mathbf{v}_{\mathbf{s}}$.

Given that the motion of the vortices is diffusive, one can make a reasonable guess for the form of $g(r, \omega)$. The mean-square displacement of a diffusing particle $\left(\left\langle x^{2}\right\rangle=2 D \tau\right)$ determines a diffusion length $r_{D}=\sqrt{2 D / \omega}$. Vortex pairs with separations less than $r_{D}$ can adiabatically follow the applied flow field and contribute to the polarizability. Pairs with separations large compared to $r_{D}$ cannot move appreciable distances before the applied field reverses direction and will therefore contribute little to the polarizability. The simplest form for $g(r, \omega)$ which has these limiting behaviors is

$$
g(r, \omega)=\frac{1}{1-i \omega \tau(r)}
$$

where $\tau(r)$ is the characteristic time for the relaxation of vortex pairs with separation $r$, i.e., $\tau(r)=r^{2} / 2 D$. Using a more rigorous treatment Ambegaokar and Teitel ${ }^{14}$ concluded that this intuitive guess is a good approximation of the true behavior if $r^{2} / 2 D$ is replaced by $r^{2} / 14 D$. [Note that the pairs with $\omega \tau(r)=1$ will dominate the imaginary part of $\epsilon(\omega)$ and therefore the dissipation.]

The expression for $\epsilon(\omega)$ can be further simplified by noting that $\alpha(r) n(r)$ is a slowly varying function compared to $g(r, \omega)$ and, therefore, $g(r, \omega)$ can be replaced within the integral of $\epsilon(\omega)$ by

$$
\begin{aligned}
& \operatorname{Re}[g(r, \omega)] \approx \Theta\left(14 D / r^{2}-\omega\right), \\
& \operatorname{Im}[g(r, \omega)] \approx(\pi r / 4) \delta(r-\sqrt{14 D / \omega}),
\end{aligned}
$$

where the step function $\Theta(x)=0$ for $x<0$ and $\Theta(x)=1$ for $x>0$. Substituting into our expression for $\epsilon(\omega)$ we obtain

$$
\begin{aligned}
& \operatorname{Re}[\epsilon(\omega)] \approx 1+4 \pi \int_{r_{0}}^{r_{D}} d \mathbf{r}^{\prime} \alpha\left(r^{\prime}\right) n\left(r^{\prime}\right)=\epsilon\left(l_{D}\right), \\
& \left.\operatorname{Im}[\epsilon(\omega)] \approx \pi^{2} r n(r) \alpha(r)\right|_{r_{D}}=\left.(\pi / 4)[d \epsilon(\omega) / d l]\right|_{l_{D}},
\end{aligned}
$$

where $\epsilon(r)$ is the dielectric function of the static theory and $l_{D}=\ln \left(r_{D} / r_{0}\right)$. We conclude from this last expression that the observed superfluid density should depend only on the response of those vortex pairs with separations less than the dynamic length scale set by the diffusion constant and that all the dissipation comes from the motion of vortex pairs at this length scale. As an example the locus of points corresponding to $l_{D}=3.5$ is shown in Fig. 1.

From Eqs. (1.29) we find that the frequency-dependent response is completely determined by the static $\epsilon(r)$. This explains why in dynamic measurements the superfluid density remains finite even above $T_{c}$. In the static theory, the disappearance of $\rho_{s}$ follows from the divergence of $\epsilon(r \rightarrow \infty)$ above $T_{c}$. However, the dynamic response $\epsilon(\omega)$ is determined by evaluating $\epsilon(r)$ at a finite length scale $l_{D}=\ln \left(r_{D} / r_{0}\right)=\frac{1}{2} \ln \left(14 D / \omega r_{0}^{2}\right)$. Since $K^{-1}(l)$ remains finite at this length scale for temperatures greater than $T_{c}$, the measured superfluid density will not vanish. Only for those higher-temperature trajectories in which $K^{-1}(l)$ diverges on length scales comparable to $l_{D}$ will $\rho_{s}$ actually vanish.

Not included in the above discussion is the dissipation caused by the free vortices. Under the action of an applied velocity field a free vortex will achieve a drift velocity given by

$$
d \mathbf{r} / d t=-\left(D / k_{B} T\right)\left(2 \pi \hbar \rho_{s 0} / m\right) \mathbf{z} \times\left(\mathbf{v}_{\mathbf{n}}-\mathbf{v}_{\mathbf{s}}\right)
$$

Once again we have neglected the terms which do not contribute to the degradation of the velocity. Because the motion of a vortex perpendicular to a flow field will decrease the flow by one unit of circulation $( \pm \mathrm{h} / \mathrm{m})$ the time rate of change of the velocity due to the motion of all the free vortices will be

$$
\begin{aligned}
d \mathbf{v}_{\mathbf{s}} / d t & =-(2 \pi \hbar / m) n \mathbf{z} \times(d \mathbf{r} / d t) \\
& =-\left(D / k_{B} T\right) n_{\text {free }}(2 \pi \hbar / m)^{2} \rho_{s 0}\left(\mathbf{v}_{\mathbf{n}}-\mathbf{v}_{\mathbf{s}}\right)
\end{aligned}
$$

where $n_{\text {free }}$ is defined in Eq. (1.18). This velocity dependent friction term can be included in the imaginary part of $\epsilon(\omega)$ by treating it as an additive contribution to $\gamma$ in Eq. (1.21). Since the relevant force is equal to $A \rho_{s 0}\left(d v_{s} / d t\right)$ we obtain 


$$
\begin{aligned}
\operatorname{Im}[\epsilon(\omega)] \approx & \left.(\pi / 4)[d \epsilon(l) / d l]\right|_{r_{D}} \\
& +n_{\text {free }} \rho_{s 0}(T)(2 \pi \hbar / m)^{2} \frac{D}{\omega k_{B} T}, \\
\operatorname{Im}[\epsilon(\omega)] \approx & \left.(\pi / 4)[d \epsilon(l) / d l]\right|_{r_{D}} \\
+ & (F / 14)(2 \pi \hbar / m)^{2}\left[\rho_{s 0}(T) / k_{B} T\right] \\
& \times \exp \left(2 l_{D}-2 l_{+}\right) .
\end{aligned}
$$

From Eq. (1.32) we see that for $T \ll T_{c}$ the dissipation will be small because $n_{\text {free }}$ is zero and the density of bound pairs with $r=r_{D}$ is small. As the temperature increases the number of vortex pairs, and in particular those with separations equal to $r_{D}$, will increase causing an increase in the dissipation. Above $T_{c}$ free vortices will also start to contribute to the total dissipation. Eventually at very high temperatures the substantial increase in the number of free vortices reduces the ability of the film to move relative to the substrate and the overall dissipation starts to decrease to zero. As a function of temperature then the dissipation of the film will be strongly peaked at a temperature above $T_{c}$ in the region where $\rho_{s}$ sharply drops to zero.

\section{Scaling properties}

As is evident from Eq. (1.15) the dependence of $K^{-1}(l)$ on temperature and film parameters is only through the integration constant $C(T)$ which is a function of $E_{c}(T) / k_{B} T$ and $\rho_{s 0}(T) / k_{B} T$. These two variables, however, are not completely independent. Because $K^{-1}(l \rightarrow \infty)=\pi / 2$ and $y(l \rightarrow \infty)=0$ at $T_{c}$, Eq. (1.12) simplifies to $C\left(T_{c}\right)=-[1+\ln (2)]$. By combining this result with Eq. (1.14) we can define a unique relationship between $\rho_{s 0}\left(T_{c}\right) / k_{B} T_{c}$ and $E_{c}\left(T_{c}\right) / k_{B} T_{c}$,

$$
\begin{aligned}
C\left(T_{c}\right)= & -[1+\ln (2)] \\
= & 2 \pi^{2} \exp \left[-2 E_{c}\left(T_{c}\right) / k_{B} T_{c}\right]-\left(\frac{\rho_{s}\left(T_{c}^{-}\right)}{\rho_{s 0}\left(T_{c}\right)}\right) \\
& +\ln \left[\frac{\rho_{s}\left(T_{c}^{-}\right)}{2 \rho_{s 0}\left(T_{c}\right)}\right] .
\end{aligned}
$$

If, in addition, we make the assumption that $\rho_{s 0}\left(T_{c}\right) / k_{B} T_{c}$ is a universal constant for helium adsorbed on a particular substrate then, independent of the film coverage, $E_{c}\left(T_{c}\right)$ will be proportional to $\rho_{s 0}\left(T_{c}\right)$. If this proportionality remains true for all temperatures below $T_{c}$ (Ref. 15) [i.e., $E_{c}(T)$ is proportional to $\rho_{s 0}(T)$ ] then it is easy to show that $K^{-1}(l)$ or equivalently $\epsilon(l)$ $=K^{-1}(l)(\hbar / m)^{2} \rho_{s 0}(T) / k_{B} T$ will be a function only of $k_{B} T / \rho_{s 0}(T)$ and $k_{B} T_{c} / \rho_{s 0}\left(T_{c}\right)$. More generally from an analysis ${ }^{16}$ of the equivalent $2 \mathrm{D}$ Coulomb-gas model one can show that the appropriate dimensionless temperature parameter becomes

$$
X=\left(T / T_{c}\right) \frac{\rho_{s 0}\left(T_{c}\right)}{\rho_{s 0}(T)} .
$$

These arguments can equally be applied to the results of the dynamic theory because the frequency-dependent dielectric constant $\epsilon(\omega)$ reduces to evaluating the static dielectric constant and its derivative at the dynamic length scale $l_{D}$ [see Eqs. (1.29) and (1.32)]. Although $\operatorname{Im}[\epsilon(\omega)]$ does contain contributions from the free vortices, $\xi_{+}$is derived from the flow equations and therefore will also depend only on the scaled temperature variable $X$. The only parameters remaining in the theory are the dynamic length scale $l_{D}$ and the constant $F$ in the free vortex density. If these parameters are either constant (independent of the temperature and film coverage) or are themselves functions of $X$ then the physically measurable quantities can be reduced to a universal scaling form,

$$
\begin{aligned}
& \frac{2 \Delta P / P}{\rho_{s 0}(T)}=\mathcal{F}_{1}\left\{X, l_{D}, F, \rho_{s 0}\left(T_{c}\right) / T_{c}\right\}, \\
& \frac{\Delta Q^{-1}}{\rho_{s 0}(T)}=\mathcal{F}_{2}\left\{X, l_{D}, F, \rho_{s 0}\left(T_{c}\right) / T_{c}\right\},
\end{aligned}
$$

where for simplicity we have assumed $l_{D}$ and $F$ to be constants. ${ }^{17}$

\section{Nonvortex excitations}

The background superfluid density $\rho_{s 0}(T)$ which determines the strength of the vortex interaction has a temperature dependence given by the nonvortex excitations of the system. In bulk liquid helium, the principal nonvortex excitations are phonons and rotons. In films, however, additional excitations are possible because of the free surface of the film and the presence of the adsorbing substrate. ${ }^{18}$ For example, Rutledge et al. ${ }^{19}$ analyze the temperature dependence of the third sound velocity with an excitation spectrum which includes surface density waves and surface rotons.

Once an excitation spectrum is chosen based on the data then to obtain the normal density $\rho_{n 0}(T)$ one follows the usual Landau prescription,

$$
\begin{aligned}
\rho_{n 0}(T) & =\rho_{s 0}(T=0)-\rho_{s 0}(T) \\
& =-\frac{1}{4 \pi \hbar^{2}} \int_{0}^{\infty} d p p^{3}\left(\frac{d n}{d \epsilon}\right),
\end{aligned}
$$

where $n=1 /\left[\exp \left(\epsilon / k_{B} T\right)-1\right]$ is the Bose distribution function and $\epsilon$ denotes the excitation energy, which is a function of the momentum $p$. As we shall see in Sec. III B, the observed scaling of $\rho_{s 0}(T)$ will constrain our choice for the nonvortex excitation spectrum.

\section{E. Calculation procedure}

\section{Static theory}

The superfluid density of the static theory can be obtained by Eq. (1.12) by noting that in the limit of $l \rightarrow \infty$ for $T<T_{c}, y(l)=0$ and $K(l \rightarrow \infty)=(\hbar / m)^{2} \rho_{s}(T) / k_{B} T$. Substituting in Eq. (1.12) we obtain,

$$
C(T)=-2\left(\frac{k_{B} T}{\pi \rho_{s}(T)(\hbar / m)^{2}}\right)+\ln \left(\frac{k_{B} T}{\pi \rho_{s}(T)(\hbar / m)^{2}}\right),
$$


which can be solved for $\rho_{s}(T)$ given $C(T) . C(T)$, in turn, can be evaluated from Eq. (1.14), i.e.,

$$
\begin{aligned}
C(T)= & 2 \pi^{2} \exp \left(-2 \frac{E_{c}\left(T_{c}\right)}{k_{B} T_{c} X}\right) \\
& -\left(\frac{T \rho_{s}\left(T_{c}^{-}\right)}{T_{c} \rho_{s 0}(T)}\right)+\ln \left(\frac{T \rho_{s}\left(T_{c}^{-}\right)}{2 T_{c} \rho_{s 0}(T)}\right),
\end{aligned}
$$

where we have used the scaling relation for the core energy,

$$
\frac{E_{c}(T)}{k_{B} T}=\frac{E_{c}\left(T_{c}\right)}{k_{B} T_{c}} \frac{T_{c} \rho_{s 0}(T)}{T \rho_{s 0}\left(T_{c}\right)}
$$

$$
b^{2}=\left.32 T_{c}[\delta C(T) / \delta T]\right|_{T_{c}}
$$

or

$$
b^{2}=\left\{1-\left.\left[T_{c} / \rho_{s 0}\left(T_{c}\right)\right]\left[\delta \rho_{s 0}(T) / \delta T\right]\right|_{T_{c}}\right\}\left[+128 \pi^{2}\left[E_{c}\left(T_{c}\right) / k_{B} T_{c}\right] \exp \left[-2 E_{c}\left(T_{c}\right) / k_{B} T_{c}\right]-64\left(\frac{\rho_{s}\left(T_{c}^{-}\right)}{\rho_{s 0}\left(T_{c}\right)}\right]+32\right]
$$

\section{Dynamic theory}

The theoretical curves presented are based on numerical integrations of Eq. (1.15) for $K^{-1}(l)$. The oscillator response is obtained by evaluating Eqs. (1.23) and (1.24) using the expressions for $\operatorname{Re}[\epsilon(\omega)]$ and $\operatorname{Im}[\epsilon(\omega)$ ] [see Eqs. (1.29) and (1.32)]. For temperatures below the static $T_{c}, n_{\text {free }}$ is set to zero and $K^{-1}(l)$ is integrated from $l=0$ to $l=l_{D}$ to obtain values for $\epsilon\left(l_{D}\right)$ and $d \epsilon\left(l_{D}\right) / d l$. Above $T_{c}$, we again numerically integrate $K^{-1}(l)$ from $l=0$ to $l=l_{D}$ to determined $\epsilon\left(l_{D}\right)$ and $d \epsilon\left(l_{D}\right) / d l$ but then the numerical integration is continued to larger values of $l$ until $y(l)=y(l=0)$. As previously discussed, we take this condition to define $l_{+}$which is then used to calculate $n_{\text {free }}$. When at some higher temperature $T_{+}$, the length scale for free vortices becomes comparable to the dynamic length scale $\left(l_{+} \approx l_{D}\right)$ the contribution to $\operatorname{Im}[\epsilon(\omega)]$ from the bound pairs is set to zero and $\operatorname{Re}[\epsilon(\omega)]$ is kept at its value at $T_{+}$. Although this assignment is somewhat arbitrary one can argue that this substitution will only have a small effect on the total response since the dissipation is already dominated by the free vortices. ${ }^{20}$ To reproduce the residual dissipation observed at low temperatures, a background dissipation can be included in the definition of $\operatorname{Im}[\epsilon(\omega)]$. In the region of $T_{c}$ this additive background dissipation will be negligible compared to the contributions from the bound pairs and free vortices.

In these calculations both $l_{D}$ and $F$ are assumed to be independent of the temperature and film coverage. ${ }^{17}$ The only other parameter is $\rho_{s 0}\left(T_{c}\right) / k_{B} T_{c}$. [Once the data are scaled $\rho_{s 0}(T=0)$ becomes an irrelevant parameter.] $C(T)$ is calculated as in the static theory.
Only $\rho_{s 0}\left(T_{c}\right) / T_{c}$ is a free parameter since the value of $E_{c}\left(T_{c}\right) / k_{B} T_{c}$ can be obtained from $\rho_{s 0}\left(T_{c}\right) / T_{c}$ from Eq. (1.33). $\rho_{s 0}(T)$ is calculated from the nonvortex excitation spectrum using Eq. (1.36).

Previous treatments often introduce a constant, denoted $b$, to describe the temperature dependence of $\rho_{s}(T)$ near $T_{c}$; i.e.,

$$
\rho_{s}(T)=\rho_{s}\left(T_{c}^{-}\right)\left[1-(b / 4) t^{1 / 2}\right],
$$

where $t$ is the reduced temperature $\left(T-T_{c}\right) / T_{c}$. By expanding both $C(T)$ and $\rho_{s}(T)$ near $T_{c}$ one can express the constant $b$ in terms of the constants $E_{c}\left(T_{c}\right) / k_{B} T_{c}$ and $\rho_{s 0}\left(T_{c}\right) / k_{B} T_{c}$,

\section{EXPERIMENTAL DETAILS}

\section{A. Apparatus}

The torsional oscillator technique originates with the work of Andronikashvili ${ }^{21}$ on the normal component of superfluid ${ }^{4} \mathrm{He}$ below the $\lambda$-transition temperature. His experimental apparatus consisted of a stack of circular disks separated by small gaps. This assembly was then placed into an aluminum cylinder and suspended from a long torsion fiber made of phosphor bronze. Because the separation between the plates was smaller than the viscous penetration depth of liquid helium at the torsional frequency $(0.04 \mathrm{~Hz})$, the period of the oscillator included contributions from the moment of inertia of the helium dragged along with the cell. By measuring changes in the period as a function of temperature, Andronikashvili was able to determine the temperature dependence of $\rho_{n}$.

The present torsional oscillator works on much the same general principles. By replacing the torsion fiber with a stiff beryllium copper ${ }^{22}(\mathrm{BeCu})$ rod we obtain a much higher natural frequency $(1275 \mathrm{~Hz})$ which facilitates signal detection and greatly improves the noise rejection. Another significant difference is the much larger $Q$ of our oscillator which makes it relatively simple to stabilize the oscillator period to better than a few parts in $10^{9}$. In addition any small dissipation within the sample can be measured by monitoring changes in the oscillator amplitude.

Figure 2 shows a schematic view of the experimental cell. An annular shell of magnesium containing a roll of Mylar $^{23}$ is attached to the $\mathrm{BeCu}$ torsion rod with a thin layer of Stycast 1266 epoxy. $^{24}$ The large diameter platform at the end of the torsion rod serves to anchor the 


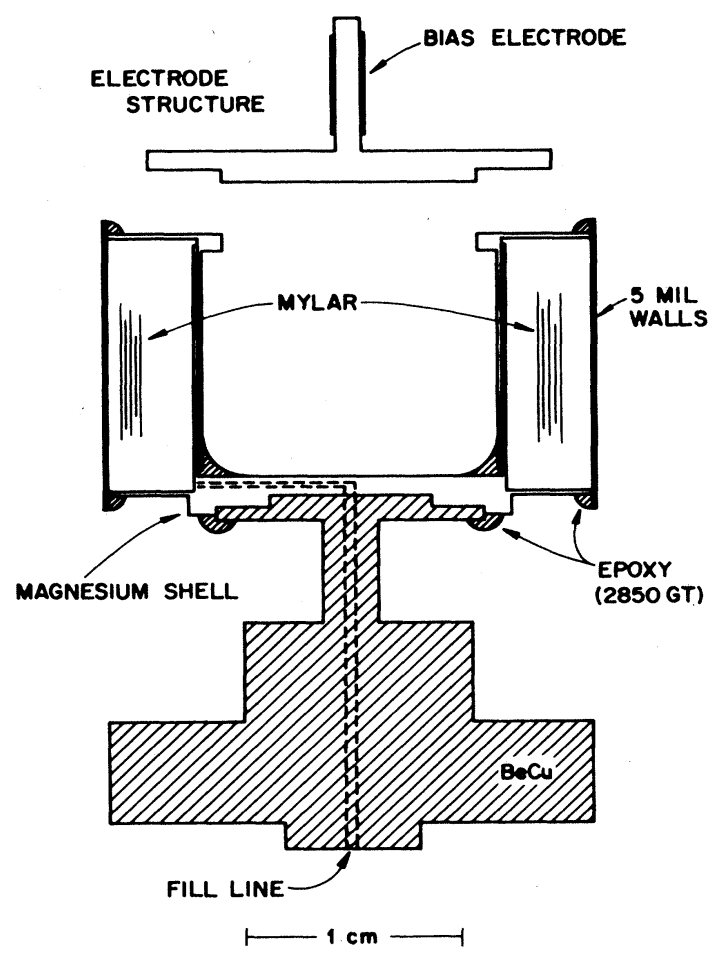

FIG. 2. Cross section of the torsional oscillator. The electrode structure is shown separated from the body of the oscillator.

magnesium shell over the largest surface area possible consistent with the constraint of a low moment of inertia. A small hole was drilled along the $\mathrm{BeCu}$ torsion rod and magnesium base for adding helium to the cell. A groove along the inner surface of the magnesium shell provides a path for the gas to flow between the Mylar layers.

Most of the structural pieces were mechanically attached with Stycast 1266 epoxy. Because of the rapid oxidation of magnesium in air a more involved procedure was required to obtain superfluid leak tight joints. Each seam was first cleaned with toluene and fine sandpaper. Stycast $2850 \mathrm{GT}$ epoxy ${ }^{24}$ was then rubbed vigorously into the seam to break the oxide coating.

The Mylar strip, $25 \mu \mathrm{m}$ thick and $1.28 \mathrm{~cm}$ wide, was wrapped onto the central spool using a coil winding machine. From adsorption studies we determine a van der Waals constant of $52 \mathrm{~K} / L^{3}$ (where $L$ denotes layer) (see the Appendix for more details). Small bubbles and imperfections can easily be seen on the surface with an optical microscope. The smallest bubbles, typically less than $2.5 \mu \mathrm{m}$ in diameter, are very numerous having an average separation of $12 \mu \mathrm{m}$ while larger bubbles $(\approx 8$ $\mu \mathrm{m})$ have typical separations of $150 \mu \mathrm{m}$. One can also find large flakes of Mylar (typically $50-100 \mu \mathrm{m}$ in diameter) on the surface.

To help affix the Mylar to the magnesium body, both the inner shell and side walls were lightly coated with a thin layer of $\mathrm{N}$ grease. ${ }^{25}$ The importance of having some means of stabilizing the Mylar layers relative to the oscillator body cannot be overemphasized. In a typical experiment the moment of inertia of the Mylar might exceed that of the helium by a factor of $10^{5}$. Therefore the period shifts caused by even microscopic motion of the Mylar layers can produce period shifts exceeding the entire period shift of the adsorbed helium film.

Subsequent layers of Mylar were added with little or no tension. To help maintain a constant average separation between the Mylar layers, a mixture of methanol and 0.3 $\mu \mathrm{m}$ alumina powder was sprayed onto each section before wrapping. The methanol eliminated much of the static cling making it easier to handle the Mylar and reduced the tendency of the layers to stick to one another on the roll. The total amount of alumina powder in the cell is estimated to have a surface area of $340 \mathrm{~cm}^{2}$.

A thin coat of $\mathrm{N}$ grease $\mathrm{e}^{25}$ was applied to the outer surface of the roll and to portions of the exposed edges to mechanically stabilize the Mylar. The second inner shell was inserted with $\mathbf{N}$ grease between it and the first inner shell. To seal the outer shell in place, all joints were first cleaned with toluene and then epoxied with $2850 \mathrm{GT}$ in the manner previously described.

The total length of the Mylar strip is $78.9 \mathrm{~m}$, giving a geometric surface area of $1.95 \mathrm{~m}^{2}$. This can be compared to a surface area of $2.09 \mathrm{~m}^{2}$ determined from an analysis of a ${ }^{4} \mathrm{He}$ isotherm made at $1.2 \mathrm{~K}$. The Mylar roll comprises $72 \%$ of the total moment of inertia which is $4.4 \mathrm{~g} \mathrm{~cm}^{2}$. The open volume of the cell, measured at room temperature, is $0.613 \mathrm{~cm}^{3}$ which is consistent with the known geometry. From the size of the annular region and the total number of layers, one calculates an average spacing of $4300 \AA$ between the layers.

The torsional motion is driven and detected by means of two capacitor electrodes at the top of the cell. A massive brass block ( $\approx 2 \mathrm{~kg}$ ) bolted to the same copper base plate as the cell supports the external electrode structure. The gap between the external and bias electrodes was set at 3-4 mil to achieve a capacitance of $1.5-2.0 \mathrm{pF}$.

The electronic feedback circuit which drives the oscillator on resonance is shown in Fig. 3. Included in the diagram are nominal values for the parameters of the feedback loop. The signal from the oscillator is first amplified by the current preamplifier of the PAR-124 lock-in amplifier $^{26}$ (LIA). The bandpass filter of the LIA is set to a small $Q$ value ( $\approx 20$ ) to avoid additional phase shifts in the feedback loop as the frequency of the oscillator changes. The amplified signal is phase shifted and then connected to the zero-crossing detector (ZCD) of the LIA reference channel. A constant amplitude sine wave signal locked to the frequency of the oscillator is then available from the reference output to drive both the oscillator and the frequency counter. Occasionally a second ZCD was inserted between the counter and the reference channel to isolate the rest of the circuit from the gating pulses of the counter. The amplitude of the oscillator is measured by recording the dc output of the LIA.

The oscillating component $\delta e^{i \omega t}$ of the distance between the electrodes is given by

$$
\delta=-\frac{A \epsilon_{0}\left|I_{\mathrm{out}}\right| e^{i \pi / 2}}{\omega V_{\mathrm{bias}} C_{p}^{2}},
$$

where $A$ is the area of the pickup electrode, $C_{p}$ its capaci- 


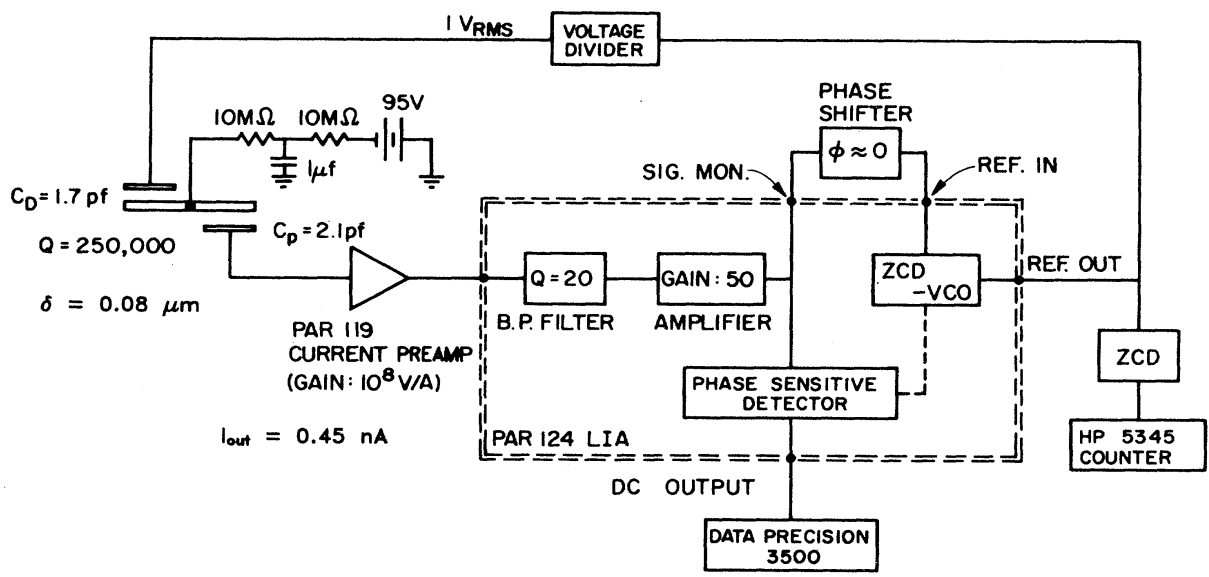

FIG. 3. Electronic feedback circuit which drives the torsional oscillator on resonance. Nominal values of the parameters of the feedback loop are shown.

tance, and $V_{\text {bias }}$ is the dc bias voltage. $\left|I_{\text {out }}\right|$ is the magnitude of the current appearing at the input of the preamplifier. The overall phase of the feedback loop is such that the drive voltage should approximately be in phase with the detected signal on resonance.

Since the oscillator was operated at constant drive, the amplitude and consequently the velocity of the substrate varies with the $Q$ of the oscillator. This variation in velocity was small even in the region of the dissipation peaks where it was about $7 \%$ of the total velocity. For the data presented the substrate velocity was approximately $0.87 \mathrm{~mm} / \mathrm{sec}$.

Nominal values for the increase (upon cooling) of the oscillator frequency from its room temperature value are $3.6 \%$ at liquid-nitrogen $\left(\mathrm{LN}_{2}\right)$ temperature and $4.2 \%$ at liquid-helium temperature. Starting with a roomtemperature value of 2000 , the $Q$ typically increased to $2 \times 10^{4}$ at $\mathrm{LN}_{2}$ temperature, eventually reaching $2 \times 10^{5}$ at $4.2 \mathrm{~K}$.

The torsional oscillator together with a vapor pressure gauge $^{27}$ were mounted on a copper platform. The entire assembly was hung from the mixing chamber of a Leyden refrigerator ${ }^{28}$ by a vibration isolator consisting of a torsion rod and spider. A 3.6-m long, 4-mil-i.d. capillary runs from the experimental cell to the $4 \mathrm{~K}$ flange where it is thermally anchored. No other heat sinks were installed in order to reduce the refluxing of helium between the torsional cell and the fill line. At the 4-K flange the fill line connects to a 10-mil-i.d. capillary which then runs up through the exchange-gas pumping line to room temperature. By judicious choices of fountain pump heater power and ${ }^{3} \mathrm{He}$-pot temperature, the stage temperature can be regulated from the lowest temperature $(\approx 35 \mathrm{mK})$ to above $2 \mathrm{~K}$. The thermometry was based on a Speer $220-\Omega$ resistor calibrated against a ${ }^{3} \mathrm{He}$ melting-curve thermometer. ${ }^{29}$ A PDI (proportional-differential-integral) regulator ${ }^{30}$ controls an electrical heater noninductively wrapped on the copper platform. The short-term temperature stability was found to be approximately $3 \mu \mathrm{K}$ at $100 \mathrm{mK}$ and $20 \mu \mathrm{K}$ at $1 \mathrm{~K}$.

With the temperature of the copper platform stabi- lized, the oscillator period requires only about $4 \mathrm{~min}$ to come into equilibrium, whereas, because of the large $Q$, 10-15 min were required for the amplitude. By averaging the period over $100 \mathrm{sec}$ the short-term stability of the period was found to be $\pm 2 \operatorname{psec}\left(\Delta P / P= \pm 3 \times 10^{-9}\right)$. The long-term drift was less than $2 \mathrm{psec} / \mathrm{h}$. The amplitude had a short-term stability of $\Delta A / A= \pm 2 \times 10^{-5}$ but could drift at rates as large as $3 \times 10^{-4} / \mathrm{h}$.

With certain sections of data it was necessary to vertically offset portions of the data because of small shifts in the period or $Q^{-1}$ caused either by the long thermal drifts in the apparatus or by the general shaking of the cryostat during helium transfers.

\section{B. Background analysis}

The measurement procedure in these experiments is conceptually quite simple. A small amount of helium gas is first added to the torsional oscillator. After annealing the sample to insure that the helium uniformly covers the Mylar substrate, the period and amplitude are measured as a function of temperature. From changes in the period of the oscillator one obtains the superfluid mass. The change in the oscillator amplitude (or $Q$ ) is a measure of the superfluid dissipation. In these low-coverage studies, the total relative change of the period $P$ and the $Q$ are quite small; $\Delta P / P \approx 5 \times 10^{-7}$ and $\Delta Q / Q \approx 2 \times 10^{-2}$. Since the temperature dependence of the oscillator itself is comparable to changes caused by the helium, it is important that the background of the oscillator be well characterized.

Previous studies ${ }^{31}$ assumed the background of the oscillator period and amplitude to depend linearly on the temperature. Closer examination of the low-temperature behavior $^{32}$ indicate a marked departure from linearity below $300 \mathrm{mK}$ as indicated in Fig. 4. Below $300 \mathrm{mK}$ the period drops below the linear behavior established at higher temperatures. Near $40 \mathrm{mK}$ the period has a minimum and then increases steadily down to the lowest temperatures examined $(\approx 5 \mathrm{mK})$. The behavior of the $Q^{-1}$ is even more striking. At high temperatures the 
$Q^{-1}$ depends linearly on the temperature down to about $500 \mathrm{mK}$ but then levels off. At $200 \mathrm{mK}$ the $Q^{-1}$ starts to decrease very rapidly; changing by a factor of two from 200 to $5 \mathrm{mK}$. Although the mechanism for this striking behavior is unknown it does appear to be an intrinsic property of the $\mathrm{BeCu}$ torsion rod. The low-temperature behavior of a number of different $\mathrm{BeCu}$ oscillators all show the same characteristic decrease in the dissipation and a minimum in the period at approximately the same temperature. ${ }^{33}$ A coin silver oscillator also exhibits these features but at a much lower temperature $(T \approx 5 \mathrm{mK}){ }^{34}$

The empty-cell background curves for the present oscillator are shown in Fig. 5 with an enlargement of the low-temperature portion of the period curve given in Fig. 6. Although we can barely define the minimum in the period with our minimum temperature, the structure in the $Q^{-1}$ is clearly observable. Above $500 \mathrm{mK}$ the period and $Q^{-1}$ have slopes of 2.80 nsec $K^{-1}$ [or $\left.(d P / d T) / P=3.57 \times 10^{-6} \mathrm{~K}^{-1}\right]$ and $3.15 \times 10^{-7} \mathrm{~K}^{-1}$, respectively. Comparisons with test oscillators made entirely of $\mathrm{BeCu}$ suggest that much of the temperature dependence in this linear region is due to the Mylar film.

In analyzing our data, we found that the background of the oscillator had changed after helium had been added to the cell. For each coverage the temperature dependence of both the period and $Q^{-1}$ in the region above the superfluid transition clearly deviates from that of the original background. This change in the background is
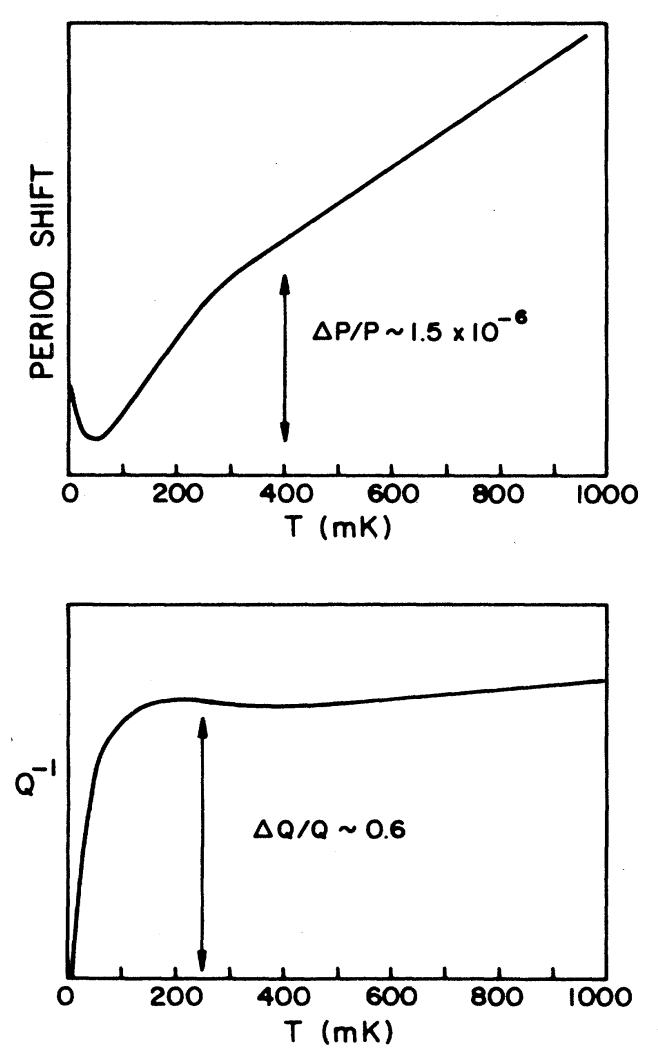

FIG. 4. Characteristic temperature dependence of the period and $Q^{-1}$ of a beryllium copper torsional element.

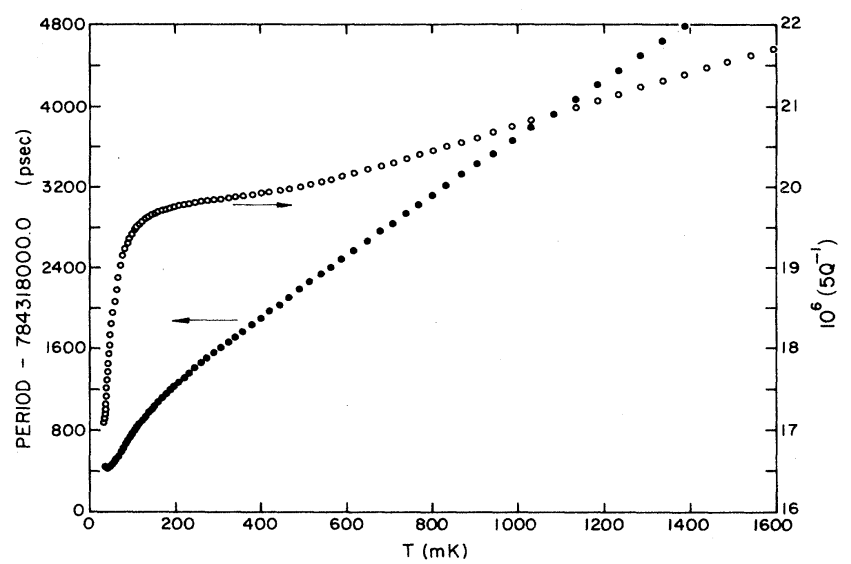

FIG. 5. Period $(\bullet)$ and $Q^{-1}(O)$ of the empty cell. The characteristic behavior of beryllium copper torsional elements is evident at our lowest temperatures.

common to each subsequent coverage and can be clearly seen when the data of different coverages are overlapped. A composite of this new background can be made by joining the nonsuperfluid regions for each coverage. The composite for the $Q^{-1}$ is plotted in Fig. 7 where the empty-cell background is also shown for comparison. The two curves agree at high temperatures $(T \approx 1.5 \mathrm{~K})$ but the composite background shows an enhanced dissipation which increases with decreasing temperature. At $200 \mathrm{mK}$ this increase is about $6 \%$. Figure 8 shows a similar comparison between the corresponding period curves which are arbitrarily matched at high temperatures. Since the composite curve represents the effective background with helium in the cell and therefore is offset from the empty cell background by a large period shift, it is not possible to determine whether the difference at the lowest temperatures is a true mass deficit. For reference, the difference between the empty cell and composite backgrounds is plotted in Fig. 9.

Because the addition of helium beyond the minimum

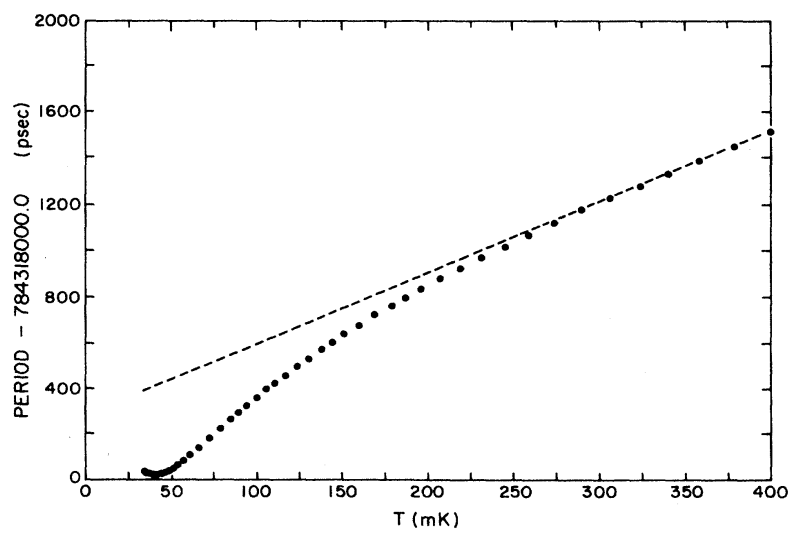

FIG. 6. Period of the empty cell for temperatures below 0.4 $\mathrm{K}$. The dashed line is an extrapolation of the linear temperature dependence observed at higher temperatures. 


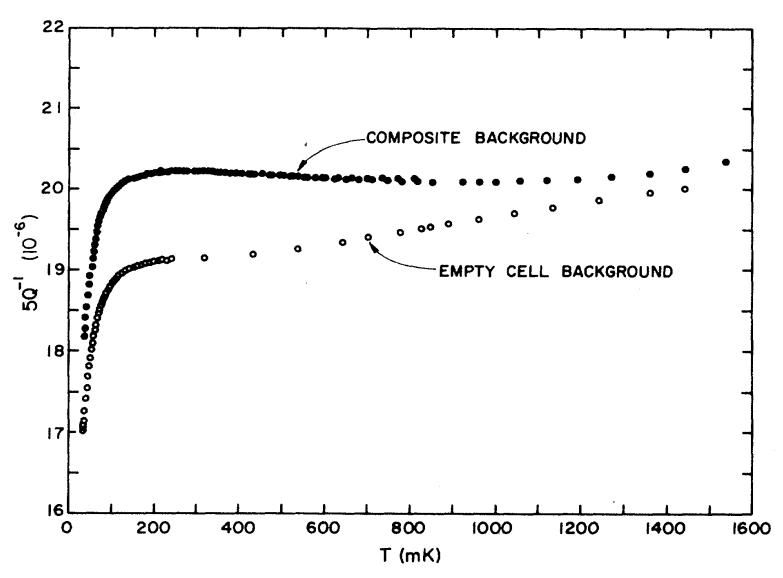

FIG. 7. Comparison between the dissipation measured with an empty cell and the dissipation determined from the composite background measured with helium in the cell. The composite background shows an enhanced dissipation which increases with decreasing temperature.

required for a superfluid transition does not strongly affect the temperature dependence of the new background, it is plausible that the shift in the background is due to the inert layer of helium adsorbed on the Mylar. In modeling the structure of a helium film, the first layers are often taken to be strongly localized by the imperfections in the substrate and the strong van der Waals attraction. Sometimes these layers are referred to as solid since the effective pressure near the substrate exceeds the solidification pressure of the bulk liquid. Subsequent layers above the inert layer are mobile and it is in these layers that superfluidity is observed.

The period shift between the two background curves at the lowest temperature corresponds to about $1.28 \mu \mathrm{M} / \mathrm{m}^{2}$ of helium or about $4.6 \%$ of the total mass in the inert layer. To explain the excess dissipation, a simple relaxation

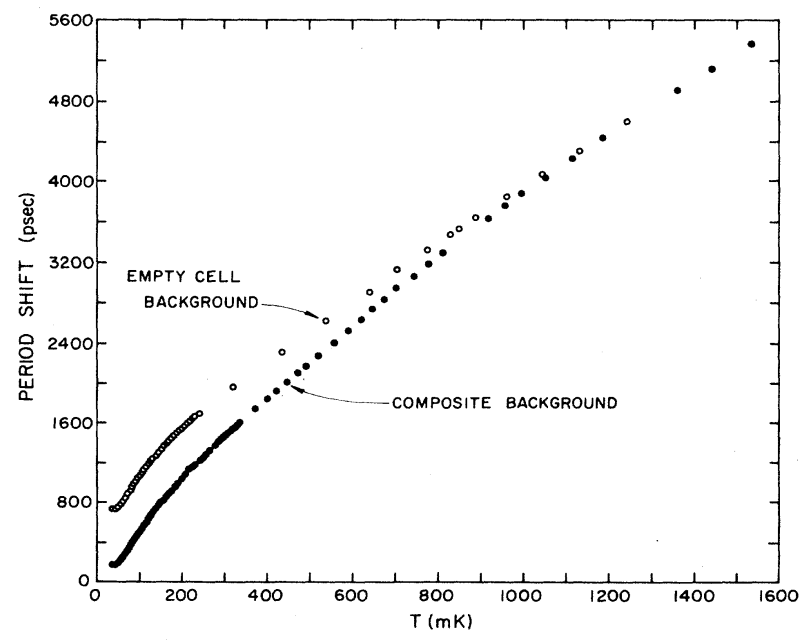

FIG. 8. Comparison between the empty-cell period shift $(\Delta P)$ and the period shift of the composite background. The composite background has been shifted vertically to match the empty cell background at high temperatures.

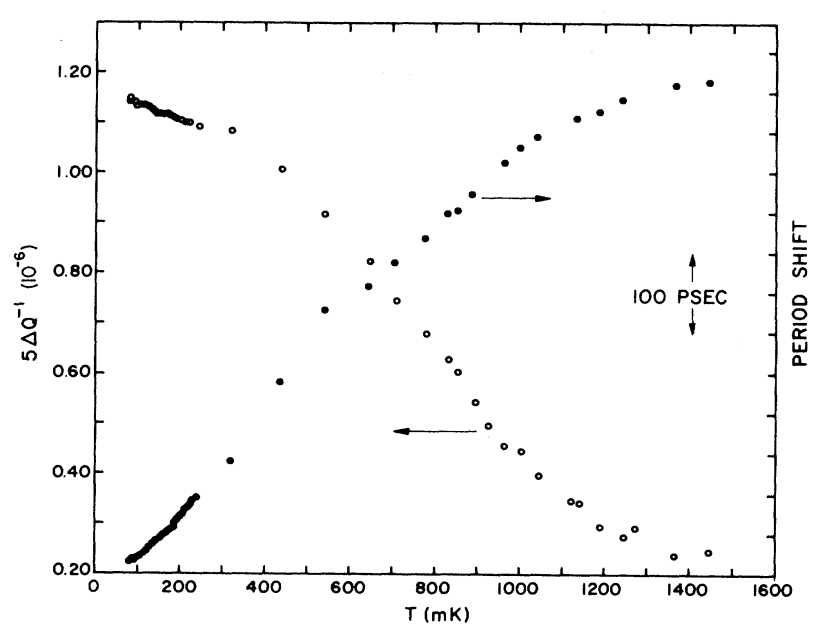

FIG. 9. The difference between the empty-cell and composite backgrounds for the period shift $(\bullet)$ and $Q^{-1}(O)$ vs the temperature. Because the origin of the period in the composite background is arbitrary, only relative period shifts are meaningful.

model would suggest characteristic relaxation times of order $10^{-4} \mathrm{sec}$, but the weak temperature dependence of the additional dissipation would argue for a broad energy distribution instead of single thermally activated process. Although studies of dislocation motion ${ }^{35}$ at large strain amplitudes in solid ${ }^{4} \mathrm{He}$ found similar behavior in the dissipation, their mechanism cannot adequately explain the period shift observed in these experiments.

For the rest of our analysis we shall assume that this anomalous background can be treated as a property of the inert layer and therefore should be subtracted from the data with the empty cell background. To make the subtraction the composite backgrounds are fitted to polynomial functions of the temperature. The difference between the fitted curves and the data is comparable to the uncertainty in the original data $\left(\triangle P / P= \pm 3 \times 10^{-9}\right.$ and $\left.\Delta Q^{-1} / Q^{-1}= \pm 5 \times 10^{-5}\right)$.

\section{Mass sensitivity}

Another important aspect of the analysis is the mass sensitivity of the oscillator. In principle this can be calculated from the moment of inertia of the cell but this method only gives results that are good to about $10 \%$. In practice it must be measured by recording the period shift as helium is added to the cell. Two such calibration runs, made at different temperatures, will be described since each has its own advantages and systematic errors.

The first calibration was done at $1.3 \mathrm{~K}$ (Fig. 10). Small amounts of helium are added to the cell and allowed to equilibrate. At these high temperatures there is a sizable gas pressure and it is not necessary (except for perhaps the first dose) to anneal the sample at higher temperatures. As helium is added the period increases until the film is sufficiently thick to become superfluid. In this normal region the slope of the period curve $(d P / d n)_{<}$versus the coverage $n$ (measured in $\mu \mathrm{M} / \mathrm{m}^{2}$ ) is a measure of the 


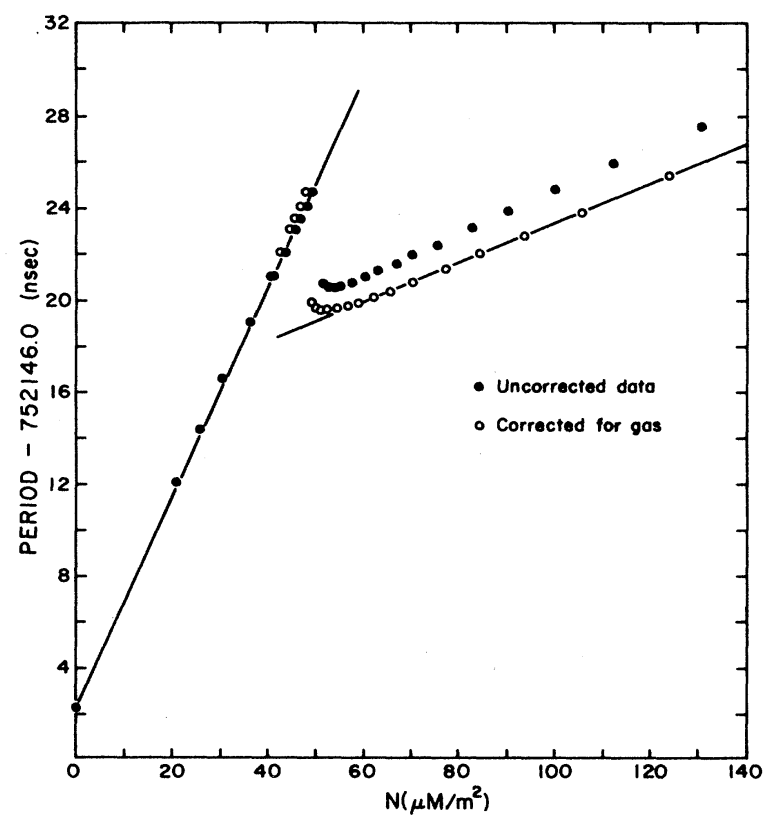

FIG. 10. A high-temperature $(T \approx 1.3 \mathrm{~K})$ calibration curve shows the period of the oscillator as helium is added to the cell. The superfluid transition occurs at approximately $50 \mu \mathrm{M} / \mathrm{m}^{2}$.

mass sensitivity of the oscillator. Once the film becomes superfluid the period drops, reflecting the decoupling of the superfluid from the motion of the substrate. As more helium is added, the period again increases but with a smaller slope. This nonzero slope indicates that a fraction of the added helium is unable to decouple from the substrate.

Macroscopic imperfections can prevent the helium from decoupling from the motion of the substrate. If the Mylar sheets were not wrapped perfectly round or were pinched together in places then the helium would move with the substrate whether or not it were superfluid. The reduction in the observable $\rho_{s}$ could also be caused by thermal excitations or by the heterogeneity of the substrate. ${ }^{36}$ Defining a phenomenological parameter $\chi$ such that $(1-\chi)$ is the fraction of the superfluid which is free to decouple we can express the period shift about the superfluid coverage by

$$
\Delta P=(d P / d n)_{<}\left[\Delta n-\Delta n(1-\chi)\left(\rho_{s} / \rho\right)\right],
$$

where $\Delta n$ is the amount of helium added. Ignoring the behavior near the onset coverage and comparing only the limiting slopes we obtain the following relationship:

$$
(d P / d n)_{>}=(d P / d n)_{<}\left[1-(1-\chi)\left(\rho_{s} / \rho\right)\right] .
$$

In order to apply Eq. (2.3) to the data in Fig. 10 we must first remove the contributions of the vapor above the film. Below the onset coverage we need only adjust the coverage for the amount of helium not physically in the cell. The gas within the cell is clamped by viscosity to the substrate owing to the small separation between the Mylar layers and therefore contributes its entire mass to the period. Above the onset coverage, however, we are only interested in the helium within the film. In this region we must subtract from the total coverage the total amount of helium in the gas. In addition the contributions of the gas to the total period must also be removed. The period shift due to the gas can be calculated from the coefficient $(d P / d n)_{<}$and the amount of gas within the cell. The size of these corrections is shown in Fig. 10.

From the slopes of the corrected data we determine the mass sensitivity to be $(d P / d n)_{<}=5.50 \times 10^{-4} \mu \mathrm{sec} /$ $\left(\mu \mathrm{M} / \mathrm{m}^{2}\right)$ and the coupling term to be $(1-\chi)\left(\rho_{s} / \rho\right)$ $=0.806$. At these coverages the films are relatively thick (ranging from 3 to 9 atomic layers) so that $\rho_{s} / \rho$ can be approximated by its corresponding value in the bulk liquid at the measuring temperature $\left(\rho_{s} / \rho=0.943\right)$. Substituting into the above expression we obtain $\chi=0.145$.

The low temperature calibration is somewhat more tedious since each addition of helium must be annealed at higher temperatures. However, we did find that once the film became superfluid it was no longer necessary to anneal subsequent coverages. Apparently the superfluid mobility is sufficiently high to uniformly distribute the helium throughout the system. Figure 11 shows the data in the superfluid region. The open circles are the period measured at $40 \mathrm{mK}$ for each coverage. The solid circles were obtained by extrapolating the high temperature (normal) background to $40 \mathrm{mK}$ using the composite background. These points correspond to the total period of the cell if the film had not become superfluid. The slope of the solid circles is $(d \dot{P} / d n)_{<}=4.18 \times 10^{-4}$ $\mu \mathrm{sec} /\left(\mu \mathrm{M} / \mathrm{m}^{2}\right)$. This value differs from that of the hightemperature calibration because of modifications made in the body of the oscillator between these two measurements. Taking the period shift into account the two coefficients agree to within $1.8 \%$.

It is interesting to note that if the fit were forced to go through the empty cell period then the value obtained for

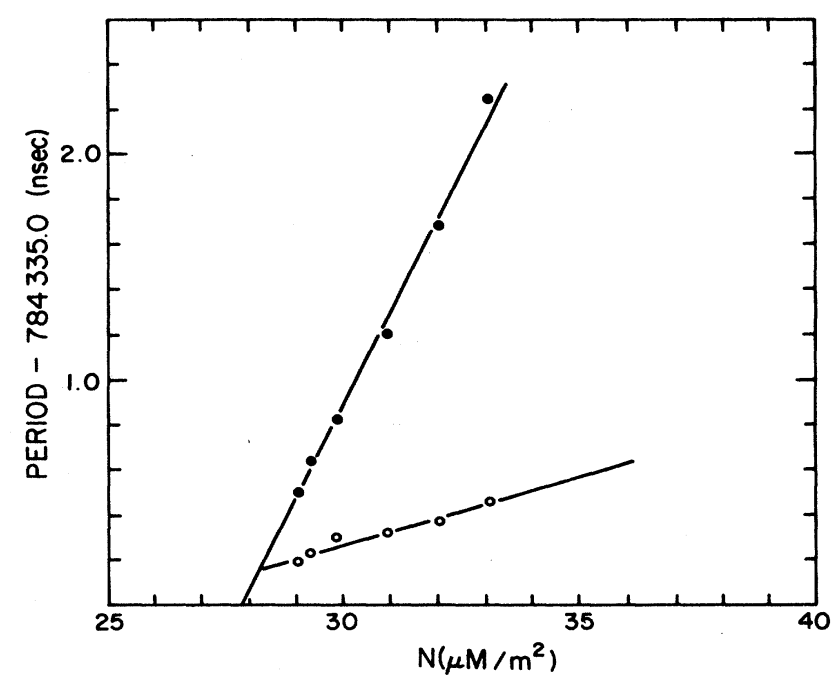

FIG. 11. Low-temperature calibration curve. The open circles indicate the oscillator period measured at $40 \mathrm{mK}$ for each coverage. The solid circles indicate the value that the oscillator period would have if the helium had not become superfluid. 
$(d P / d n)_{<}$would be smaller. This might suggest that initial helium doses contribute less than their full mass to the oscillator. This deficit in mass is in fact comparable in size to the mass difference between the empty cell and composite backgrounds (see Fig. 9).

From an analysis of the low temperature superfluid data, we determine $(1-\chi)\left(\rho_{s} / \rho\right)=0.856$. If we take $\rho_{s} / \rho$ equal to one, we find $\chi$ to be 0.144 in agreement with the value obtained at high temperatures. The fact that the film thicknesses in the two calibrations are very different (greater than four layers in the high-temperature calibration compared to the 0.10 to 0.40 active superfluid layers in the low-temperature calibration) strongly suggests that the hindrance factor must be predominantly caused by the geometry of the Mylar roll.

To obtain the coefficient $A / M$ introduced in Eqs. (1.23) and (1.24) we normalize the mass sensitivity by the total period; i.e., $A / M=(2 / m)\left[(1 / P)(d P / d n)_{<}\right]$where $m$ is the molar mass of ${ }^{4} \mathrm{He}$. Substituting $(d P / d n)_{<}$from above we obtain $A / M=0.266 \mathrm{~m}^{2} / \mathrm{g}$.

\section{Nonlinear behavior}

In previous measurements ${ }^{37}$ as the drive level or substrate velocity was increased the dissipation peaks became broader and were shifted to lower temperatures. To quantify these effects at lower coverages, we have made a series of temperature sweeps at different drive levels using a film coverage of $33.1 \mu \mathrm{M} / \mathrm{m}^{2}\left(T_{\text {peak }}=379\right.$ $\mathrm{mK})$.

The procedure for taking data was slightly different from the one previously described. Instead of regulating the experimental stage at each temperature, we imposed a slow temperature drift by introducing a ramping voltage to the signal input of the temperature regulator. The drift rate was sufficiently slow to avoid systematic errors due to the relaxation time (or ringdown time) of the oscillator. Although a temperature hysteresis of a few $\mathrm{mK}$ was observed, the widths of the dissipation peaks match those measured in previous runs. A typical drift rate was about $50 \mu \mathrm{K} / \mathrm{sec}$.

The sequence of data is shown in Fig. 12. Each curve is marked with the substrate velocity (in units of $\mathrm{mm} / \mathrm{sec}$ ) calculated using Eq. (2.1). The $Q^{-1}$ data are presented with no background subtracted since in this temperature region the background $Q^{-1}$ is only weakly temperature dependent compared to the dissipation in the film.

As is evident by the vertical displacement between the curves, the background dissipation increases with the oscillator amplitude. The background dissipation also showed hysteresis. Upon returning to the nominal drive level $(0.087 \mathrm{~cm} / \mathrm{sec})$ the background dissipation had increased approximately $25 \%$ over its original value. This excess dissipation eventually decayed away with a time constant of a few weeks.

The data can be divided into two distinct velocity regions. Below $1 \mathrm{~cm} / \mathrm{sec}$, the dissipation peaks share the same characteristic shape but increase in size and broaden with increasing drive level. The dissipation "peaks" for velocities greater than $1 \mathrm{~cm} / \mathrm{sec}$ have a more gentle temperature dependence on both sides of the peak.

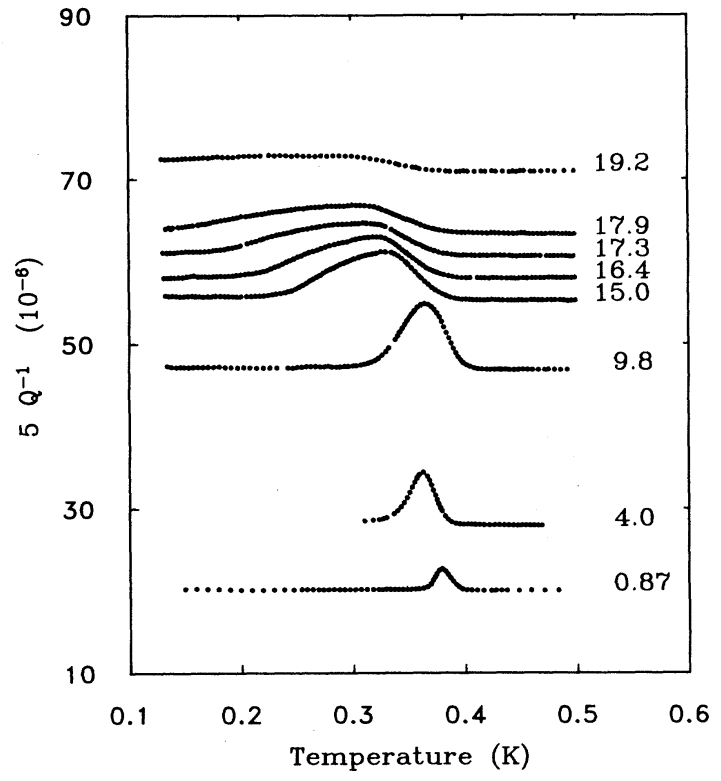

FIG. 12. Velocity dependence of the dissipation peak for coverage no. 5. The nominal substrate velocities (in units of $\mathrm{mm} / \mathrm{sec}$ ) are indicated for each trace.

The dissipation maxima are also shifted to much lower temperatures. Although the peaks continue to broaden with increasing drive, the height of the peaks decreases.

In Fig. 13, we plot the width of each peak measured at half the maximum dissipation as a function of the velocity. Although this measure is somewhat inappropriate for the higher drive traces it does provide a guide to the overall behavior. We find that the velocity scale for nonlinear behavior is comparable to that observed in thicker films. In our previous studies of a film with $T_{\text {peak }} \approx 1.3 \mathrm{~K}$, the width of the dissipation peak increased only by $10 \%$ at a substrate velocity of $0.5 \mathrm{~cm} / \mathrm{sec}$. In addition the dissipation maximum did not change appreciably over the range examined; 0.015 to $0.5 \mathrm{~cm} / \mathrm{sec}$. We conclude from these measurements that the linear region extends to much higher velocities than initially reported by Bishop ${ }^{37}$ and that these nonlinear effects become more pronounced at lower coverages.

As is indicated by Fig. 13 our nominal substrate velocity of $0.87 \mathrm{~mm} / \mathrm{sec}$ does not put the data strictly in the linear region. This causes the transition region and in particular the dissipation peaks to be broader. Fortunately this broadening is predominantly on the lowtemperature side of the peak and therefore one can still make useful comparisons with the theoretical predictions by stressing the high-temperature side of the peaks. As we shall see later this will be an important correction to the analysis.

An estimate of the substrate velocity required for nonlinear effects to become important can be made by examining the effect of an external flow field on the stability of a bound pair. In the presence of an external flow the interaction energy of a bound pair [Eq. (1.3)] is reduced by a term proportional to the velocity, 


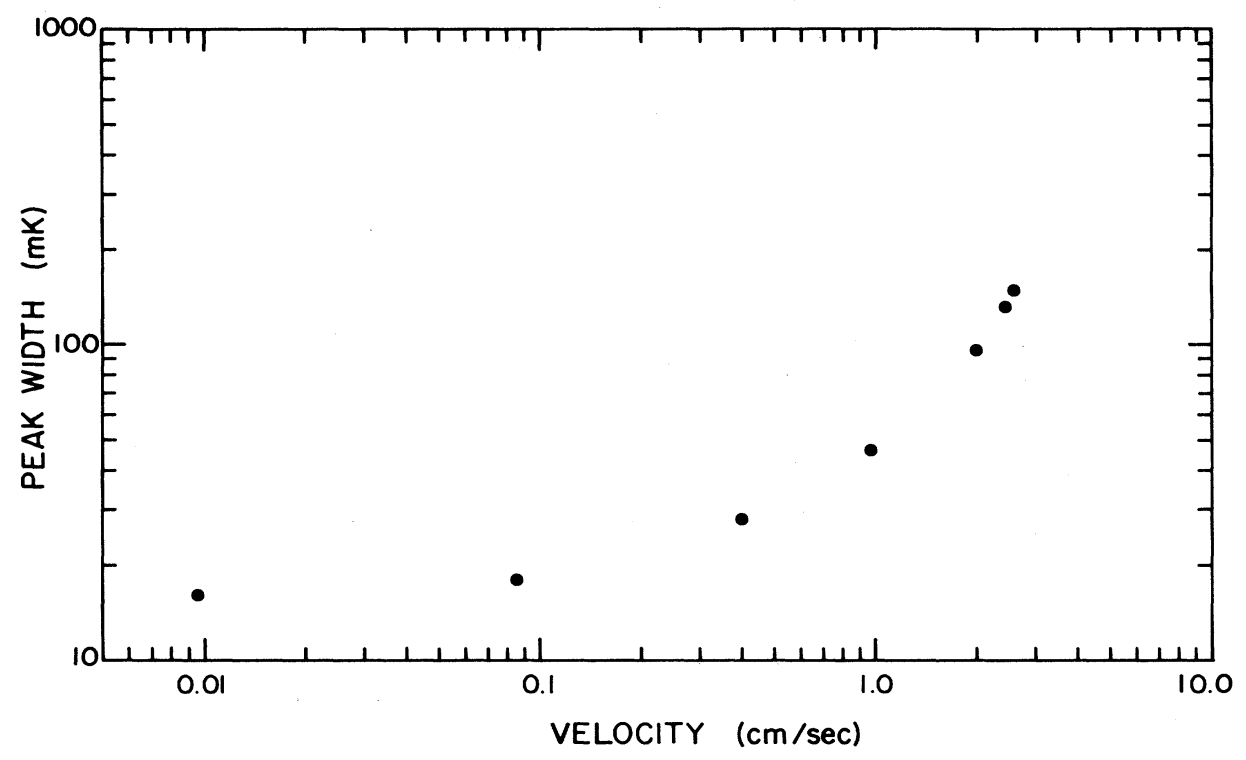

FIG. 13. Width of the dissipation peaks of Fig. 12 plotted against the substrate velocity.

$$
\begin{aligned}
E_{\text {pair }}^{\prime}= & 2 \pi n^{2} K_{0} \epsilon^{-1}(r) \ln \left(r / r_{0}\right)+2 E_{c}(T) \\
& -\left[(h / m) \rho_{s 0}(T)\right] \mathbf{v}_{\mathbf{s}} \cdot \mathbf{r},
\end{aligned}
$$

where the factor of $\epsilon(r)$ is inserted to include the effects of the smaller vortex pairs. For fixed $v_{s}$, Eq. (2.4) indicates that there exists a critical separation $r_{c}$ beyond which the pair is no longer energetically bound. Solving for $r_{c}$ we obtain

$$
r_{c} \approx \frac{(\hbar / m)}{\epsilon\left(r_{c}\right) v_{s}}
$$

Clearly even vortex pairs with separations less than $r_{c}$ are only metastable. AHNS estimate the steady-state density of free vortices induced by the presence of the velocity field in the small velocity limit to be

$$
n_{\text {free }}=\left(\frac{y\left(l_{c}\right)}{r_{c}^{2}}\right)\left[2 \pi K^{-1}\left(l_{c}\right)\right]^{1 / 2} e^{\pi K\left(l_{c}\right)},
$$

where $l_{c}=\ln \left(r_{c} / r_{0}\right)$. The induced $n_{\text {free }}$ is therefore proportional to the density of vortex pairs with a separation equal to the critical value $r_{c}$. Although vortex pairs of other separations also contribute to $n_{\text {free }}$, the pairs at this critical separation dominate the dissociation rate.

In our experiment the velocity field is oscillatory and therefore $r_{c}$ will alternately move in from infinity $\left(v_{s}=0\right)$ to its minimum value when $v_{s}$ is at a maximum. When $v_{s}$ is sufficiently large for $r_{c}$ to become comparable to $r_{D}$ we should expect to see nonlinear effects developing. From our data we estimate the critical value of $v_{s}$ to be greater than $0.01 \mathrm{~cm} / \mathrm{sec}$ which would correspond to a diffusion length $l_{D}$ less than 13 .

In a more detailed analysis of the nonlinear behavior, Gillis et $a l .{ }^{38}$ have included the effects of an external velocity field within the recursion equations of the vortex pairs. From these modified equations for $y(l)$ and $K^{-1}(l)$, Adams and Glaberson ${ }^{17}$ have calculated the theoretical dissipation for their torsional oscillator over a range of substrate velocities. Their results exhibit many of the same general features as our data. A more detailed comparison with our data is in progress.

\section{ANALYSIS}

\section{A. General observations}

In searching for systematic behavior in the data it is often convenient to use the temperature of the dissipation peak, $T_{\text {peak }}$, since it is an unambiguous signature of the transition. In Fig. 14 we plot $T_{\text {peak }}$ versus coverage for the data tabulated in Table I. Films with higher transition temperatures have been included to place the low coverage data in perspective. For temperatures above 1.1 $K$ the coverage has been corrected for the helium in the vapor. Two distinct regions are readily apparent from the data. From 0.9 to $1.4 \mathrm{~K}, T_{\text {peak }}$ can be locally approximated by a straight line with a slope of 42.8 $\mathrm{mK} /\left(\mu \mathrm{M} / \mathrm{m}^{2}\right)$. Below $0.8 \mathrm{~K}, T_{\text {peak }}$ first decreases much more rapidly with decreasing coverage but then decreases more gradually as $T=0$ is approached. By extrapolating the data to $T=0$, we estimate the nonsuperfluid layer to be $27.85 \mu \mathrm{M} / \mathrm{m}^{2}$. Taking the density of this layer to be that of the bulk solid $\left(\rho_{\text {solid }}=0.191 \mathrm{~g} / \mathrm{cm}^{2}\right)$, this coverage corresponds to a thickness of $5.8 \AA$ or about 1.6 atomic layers (one atomic layer is arbitrarily taken to be $3.6 \AA$ ). The conversion of coverage into layers in Fig. 14 assumes that subsequent layers of helium have a density given by the bulk liquid $\left(\rho_{\text {liq }}=0.145 \mathrm{~g} / \mathrm{cm}^{2}\right)$. (A more detailed description of the film thickness determination can be found in the Appendix.) 


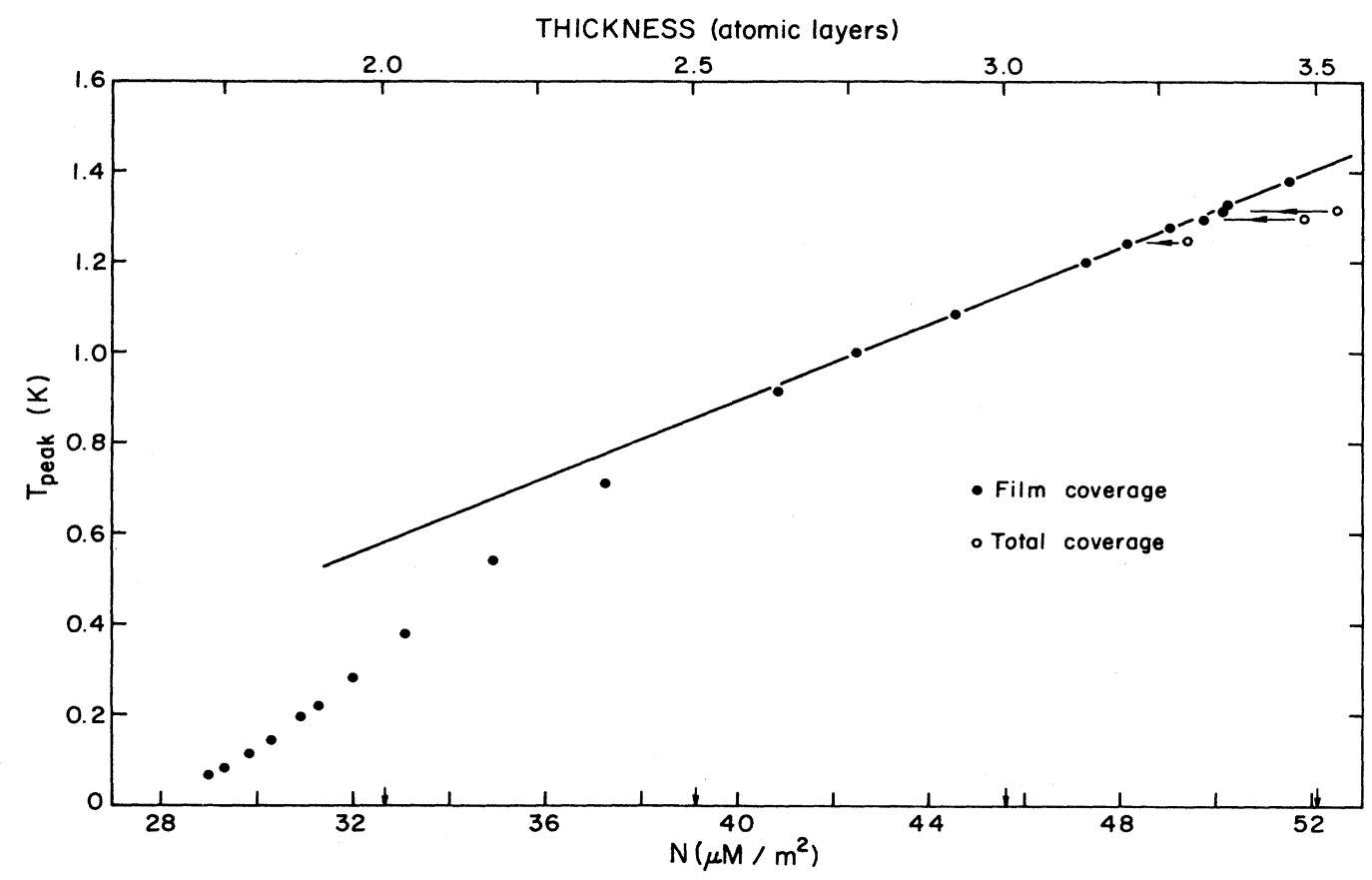

FIG. 14. Variation of $T_{\text {peak }}$ with film coverage. The line is drawn to emphasize the coverage dependence of the lower coverage data. The higher coverages have been corrected for depletion by the gas. The nominal thickness in atomic layers is shown.

This break in the coverage dependence of $T_{\text {peak }}$ may indicate a modification in the normal density of the helium film. In our experiment, this change occurs approximately at one atomic layer above the nonsuperfluid layer. We conjecture that as the superfluid layer exceeds one layer, new degrees of freedoms or modes are possible for the system. With the appearance of these low energy excita-

TABLE I. Helium film coverage and $T_{\text {peak }}$ data.

\begin{tabular}{ccr}
\hline $\begin{array}{c}N_{\text {total }} \\
\left(\mu \mathrm{M} / \mathrm{m}^{2}\right)\end{array}$ & $\begin{array}{c}N_{\text {corrected }} \\
\left(\mu \mathrm{M} / \mathrm{m}^{2}\right)\end{array}$ & $\begin{array}{r}T_{\text {peak }} \\
(\mathrm{mK})\end{array}$ \\
\hline 28.97 & 28.97 & 63.5 \\
29.03 & 29.03 & 67.0 \\
29.32 & 29.32 & 82.8 \\
29.85 & 29.85 & 114.3 \\
30.30 & 30.30 & 146.2 \\
30.91 & 30.91 & 192.4 \\
32.00 & 32.00 & 281.8 \\
33.09 & 33.09 & 379.3 \\
34.92 & 34.92 & 539.2 \\
37.25 & 37.25 & 710.0 \\
40.88 & 40.88 & 914.1 \\
42.49 & 42.49 & 999.0 \\
44.55 & 44.55 & 1086.8 \\
49.43 & 48.15 & 1242.0 \\
51.85 & 49.74 & 1297.2 \\
52.56 & 50.16 & 1312.5 \\
50.41 & 47.31 & 1201.8 \\
52.61 & 48.15 & 1240.0 \\
55.43 & 49.07 & 1281.0 \\
59.68 & 50.24 & 1329.7 \\
65.35 & 51.56 & 1380.9 \\
\hline \hline
\end{tabular}

tions the transition temperature occurs at a lower temperature than would have been expected based on the trend established by lower coverages.

To avoid possible complications inherent in the thicker films, we examine in detail only those films with coverages less than one active superfluid layer. Table II lists the parameters for these data. We have defined the active layer coverage $N_{\text {active }}$ to be that coverage in excess of the nonsuperfluid layer as determined from the extrapolation described above. The average separation between atoms is calculated assuming a closed-packed structure.

The period and amplitude data for these coverages are shown in Figs. 15 and 16. The period data for each coverage have been shifted vertically to emphasize the composite background described in Sec. II B. As is evident from the relative size of the background compared to the superfluid signals, the background determination is a significant source of the systematic error.

The normalized data with the background subtracted are plotted in Figs. 17 and 18. For historical reasons a factor of 5 is included in the presentation of the $\Delta Q^{-1}$ data to facilitate a common scale between the reduced period and the $\Delta Q^{-1}$.

The qualitative features of the reduced data resemble those obtained for much thicker films $\left(T_{c}>1 \mathrm{~K}\right)$. The reduced period or superfluid mass rapidly decreases to zero in the temperature region of the dissipation peak. Below the region of the transition, the superfluid mass has a more gradual temperature dependence and approaches its $T=0$ value with zero slope. (This is particularly evident in the data of the two highest coverages.) The anomalous curvature in the reduced period and dissipation below 75 $\mathrm{mK}$ is an artifact of the composite background. Since no 
TABLE II. Film parameters of low coverage data. The transition temperatures listed were estimated from the value of $\rho_{s 0}\left(T_{c}\right) / T_{c}$ and $\rho_{s 0}(T)$ for each coverage (see the text).

\begin{tabular}{rccccrr}
\hline & $\begin{array}{c}\text { Coverage } \\
\left(\mu \mathrm{M} / \mathrm{m}^{2}\right)\end{array}$ & $\begin{array}{c}N_{\text {active }} \\
\left(\mu \mathrm{M} / \mathrm{m}^{2}\right)\end{array}$ & $\begin{array}{c}\text { Area/atom } \\
\left(\AA^{2}\right)\end{array}$ & $\begin{array}{c}\text { Average } \\
\text { separation } \\
(\AA)\end{array}$ & $\begin{array}{c}T_{\text {peak }} \\
(\mathrm{mK})\end{array}$ & $\begin{array}{c}T_{c} \\
(\mathrm{mK})\end{array}$ \\
\hline 1 & 29.03 & 1.17 & 141.6 & 12.8 & 67.1 & 65.1 \\
2 & 29.85 & 2.00 & 83.0 & 9.8 & 114.3 & 107.7 \\
3 & 30.91 & 3.06 & 54.3 & 7.9 & 192.4 & 183.8 \\
4 & 32.00 & 4.15 & 40.0 & 6.8 & 281.8 & 272.5 \\
5 & 33.09 & 5.24 & 31.7 & 6.1 & 379.3 & 370.7 \\
\hline \hline
\end{tabular}

data were available with transition temperatures less than $75 \mathrm{mK}$, the composite background had to be extrapolated to lower temperatures by using the empty cell background. This procedure can lead to sizable errors since both the period and $Q^{-1}$ are strongly temperature dependent below $50 \mathrm{mK}$.

The Nelson and Kosterlitz ${ }^{12}$ prediction for $\rho_{s}\left(T_{c}^{-}\right)$ [Eq. (1.17)], indicated by the line marked $\chi=0$ in Fig. 17, represents the maximum observable superfluid density for a given transition temperature. In practice this value is reduced by the geometrical hindrance of the Mylar roll. The value of $\rho_{s}\left(T_{c}^{-}\right)$appropriate for our experimental cell is indicated by the line $\chi=0.145$. Surprisingly the $\chi=0$ line appears to be in better agreement with the data since it passes through the knee of each period curve. However, one must remember that, in the dynamic response theory, the rapid decrease in the period is shifted to a higher temperature than the static $T_{c}$. In addition the position of the knee in the period curve will overestimate the value of $\rho_{s}\left(T_{c}^{-}\right)$. This illustrates how misleading qualitative comparisons can be. Only detailed comparisons with calculations based on the dynamic theory are reliable.

The coverage dependence of the dissipation peaks in Fig. 18 exhibits an interesting nonsystematic behavior. The dissipation peak of the lowest coverage (average

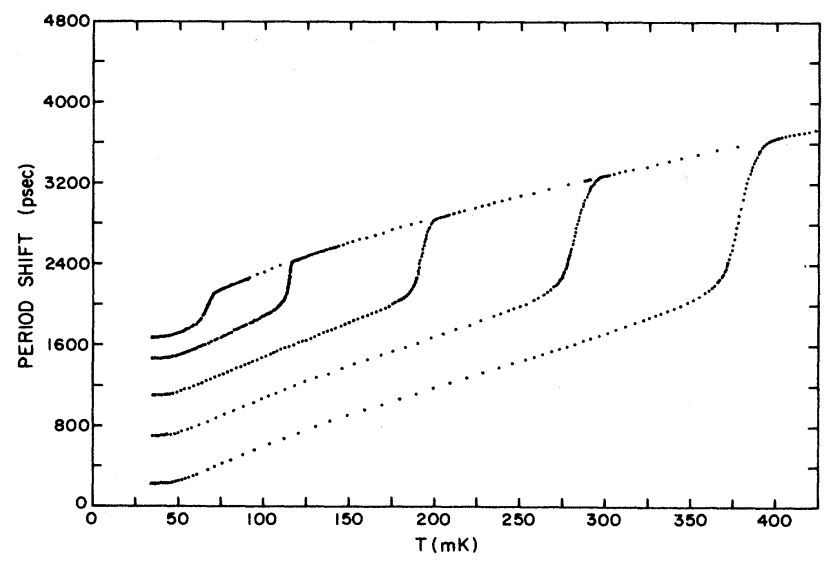

FIG. 15. Period data for five different coverages. Each period curve has been shifted vertically to illustrate the composite background. The temperature dependence in the normal regions above the superfluid transitions is found to be coverage independent. atomic separation $\approx 13 \AA$ ) is relatively broad with an asymmetric tail extending to lower temperatures. At this low coverage, the helium film is probably very sensitive to the imperfections in the Mylar substrate and is therefore spatially inhomogeneous. Increasing the coverage slightly (coverage no. 2) dramatically sharpens the dissipation peak consistent with our expectations that higher coverages would be less sensitive to imperfections in the substrate. The behavior of the next three coverages, however, contradict this simple explanation as they all exhibit small and broad peaks. Because these peaks are much more symmetric about $T_{\text {peak }}$ we believe that this broadening has a different origin than the broadening observed for the lowest coverage. Overall, these dissipation peaks, including that of coverage no. 2 , are significantly broader when compared with the dissipation peaks of films with $T_{\text {peak }}>1 \mathrm{~K}$. For example the relative width of the dissipation peak of coverage no. 5 is $\Delta T / T_{\text {peak }} \approx 0.04$, whereas for a much thicker film $\Delta T / T_{\text {peak }}$ is less than 0.003 .

Another interesting feature of the data is the residual dissipation found below $T_{\text {peak }}$. This dissipation is also observed in higher coverage data but is not explained by the AHNS theory. Within the accuracy of our measurements the residual dissipation is the same for each coverage. Although this dissipation is temperature dependent

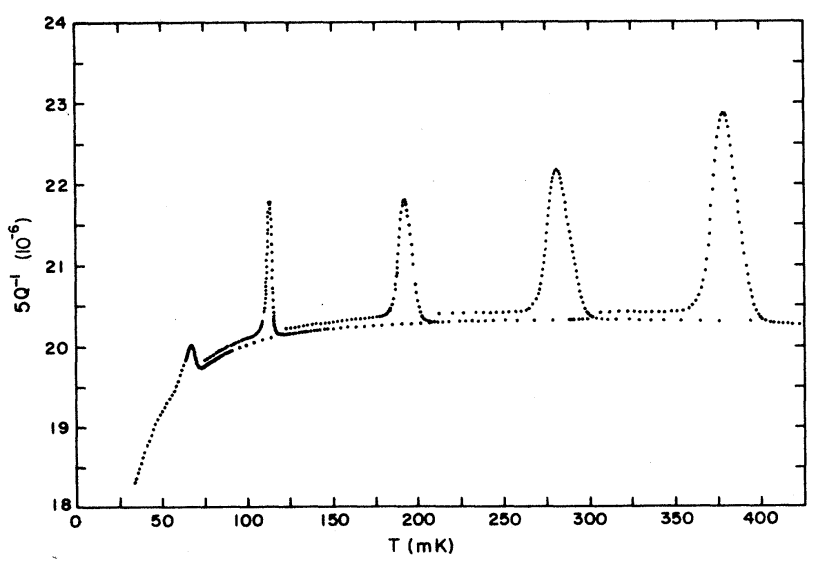

FIG. 16. $Q^{-1}$ data for five different coverages. As in the period data, the normal regions of the different coverages overlap each other. 


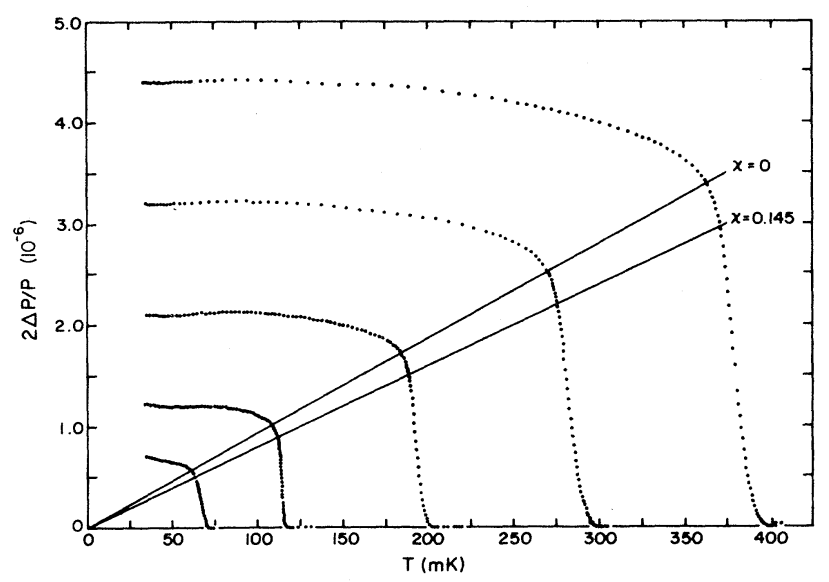

FIG. 17. Reduced period data. $2 \Delta P / P$, which is proportional to the superfluid density $\rho_{s}(T)$, is obtained by subtracting the composite background from the original data and normalizing by the total period. The solid lines represent the KosterlitzNelson prediction for $\rho_{s}\left(T_{c^{-}}\right)$assuming two different values of the geometric hindrance $\chi$.

it appears to be independent of the superfluid coverage of the film. ${ }^{39}$

To explain this residual dissipation Browne and Don$\operatorname{tach}^{40}$ postulate a finite population of unpaired free vortices pinned to surface imperfections in the film. These vortices can be depinned by thermal fluctuations or external flow fields and thereby act as a reservoir of free vortices even below $T_{c}$. Once depinned, these vortices diffuse throughout the film until they encounter another pinning site. Just like the thermally excited free vortices, these vortices destroy superfluid flow and consequently contribute to the overall dissipation. An estimate based on Eq. (1.32) indicates that only 400 free vortices per $\mathrm{cm}^{2}$ would be needed to produce the observed dissipation.

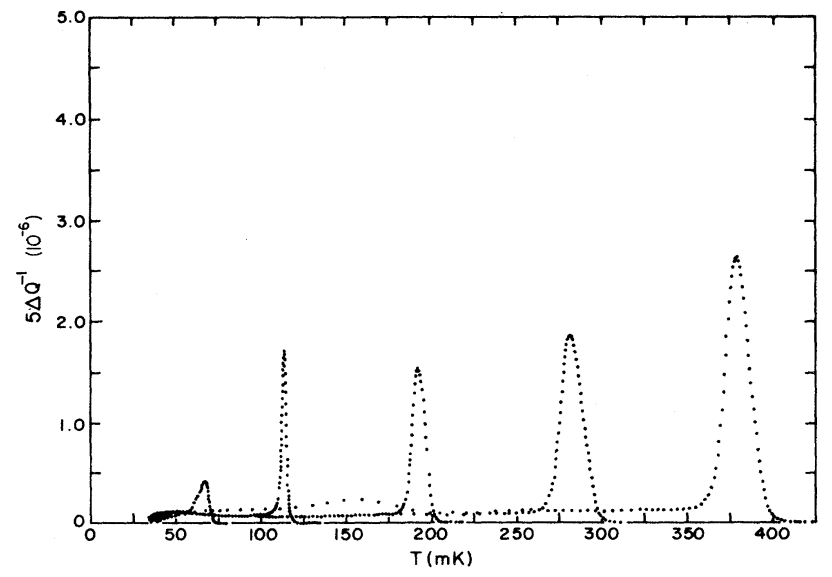

FIG. 18. Reduced $Q^{-1}$ data. The broad peak near $150 \mathrm{mK}$ belongs to the data of coverage no. 5 and is thought to be a third sound resonance. The dissipation below each dissipation peak appears to follow a universal curve independent of the coverage.
But in order for the dissipation to be coverage independent this free vortex density times the vortex diffusivity would have to vary inversely with the superfluid density. In addition, this model does not explain the observed linear dependence of the dissipation on the temperature.

\section{B. Comparisons with dynamic theory}

To facilitate comparisons between data of different coverages we can take advantage of the predicted scaling behavior. As discussed previously in Sec. I C, $2 \Delta P / P$ and $\Delta Q^{-1}$, when scaled by $\rho_{s 0}(T)$, should exhibit universal behavior when expressed as functions of the reduced temperature variable $X=\left(T / T_{c}\right)\left[\rho_{s 0}\left(T_{c}\right) / \rho_{s 0}(T)\right]$, i.e.,

$$
\begin{aligned}
& \frac{2 \Delta P / P}{\rho_{s 0}(T)}=\mathscr{F}_{1}\left\{X, l_{D}, F, \rho_{s 0}\left(T_{c}\right) / T_{c}\right\}, \\
& \frac{\Delta Q^{-1}}{\rho_{s 0}(T)}=\mathscr{F}_{2}\left\{X, l_{D}, F, \rho_{s 0}\left(T_{c}\right) / T_{c}\right\},
\end{aligned}
$$

where the parameters $l_{D}, F$, and $\rho_{s 0}\left(T_{c}\right) / T_{c}$ are expected to be substrate dependent but are assumed not be coverage dependent.

When applying the above scaling relations we can avoid the necessity of determining $\rho_{s 0}(T)$ by replacing $\rho_{s 0}(T)$ with its value at $T_{c}$ to obtain a scaling relation that will be valid only near $T_{c}$. In addition since the static $T_{c}$ does not correspond to any identifiable feature in the data it is convenient to replace $T_{c}$ by the temperature of an unambiguous feature of the data which scales appropriately with $T_{c}$. The obvious choice is $T_{\text {peak }}$. This substitution can be justified because the ratio $T_{\text {peak }} / T_{c}$ is uniquely determined by the universal scaling functions Eq. (3.1) for fixed values of the parameters. Similarly one can replace $\rho_{s 0}\left(T_{c}\right)$ by $T_{\text {peak }}$ on the left-hand side of the equation since $\rho_{s 0}\left(T_{c}\right) / T_{c}$ is assumed to be a constant. With these modifications the scaling relations become

$$
\begin{aligned}
& \frac{2 \Delta P / P}{T_{\text {peak }}}=\mathcal{H}_{1}\left\{T / T_{\text {peak }}, l_{D}, F, \rho_{s 0}\left(T_{c}\right) / T_{c}\right\}, \\
& \frac{\Delta Q^{-1}}{T_{\text {peak }}}=\mathcal{H}_{2}\left\{T / T_{\text {peak }}, l_{D}, F, \rho_{s 0}\left(T_{c}\right) / T_{c}\right\} .
\end{aligned}
$$

In Fig. 19 we show the scaled data $2 \Delta P / P T_{\text {peak }}$ and $\Delta Q^{-1} / T_{\text {peak }}$ plotted versus $T / T_{\text {peak }}$ for each coverage. To emphasize the scaling behavior, the data for each coverage are superimposed in Fig. 20. The data in the transition region are shown with an expanded scale. (For clarity the data of coverage no. 1 are omitted.)

Except for the data of coverage no. 2 the period and dissipation data appear to follow universal curves in the region of the transition. The universal behavior in the period shift, however, appears to extend to much lower temperatures than one would have predicted. In fact, the minor deviations which are observed between the different coverages can be attributed to systematic errors inherent in the background subtraction and to the coupling of the oscillator resonance to third sound modes. [A clear example of a third sound resonance can be seen (Fig. 19) in the dissipation data of coverage no. 5 near $\left.T / T_{\text {peak }}=0.5.\right]$ 
This low temperature scaling is interesting because it cannot be a consequence of the vortices. At low temperatures, the density of vortex pairs should be exponentially small since the minimum energy to create a vortex pair is

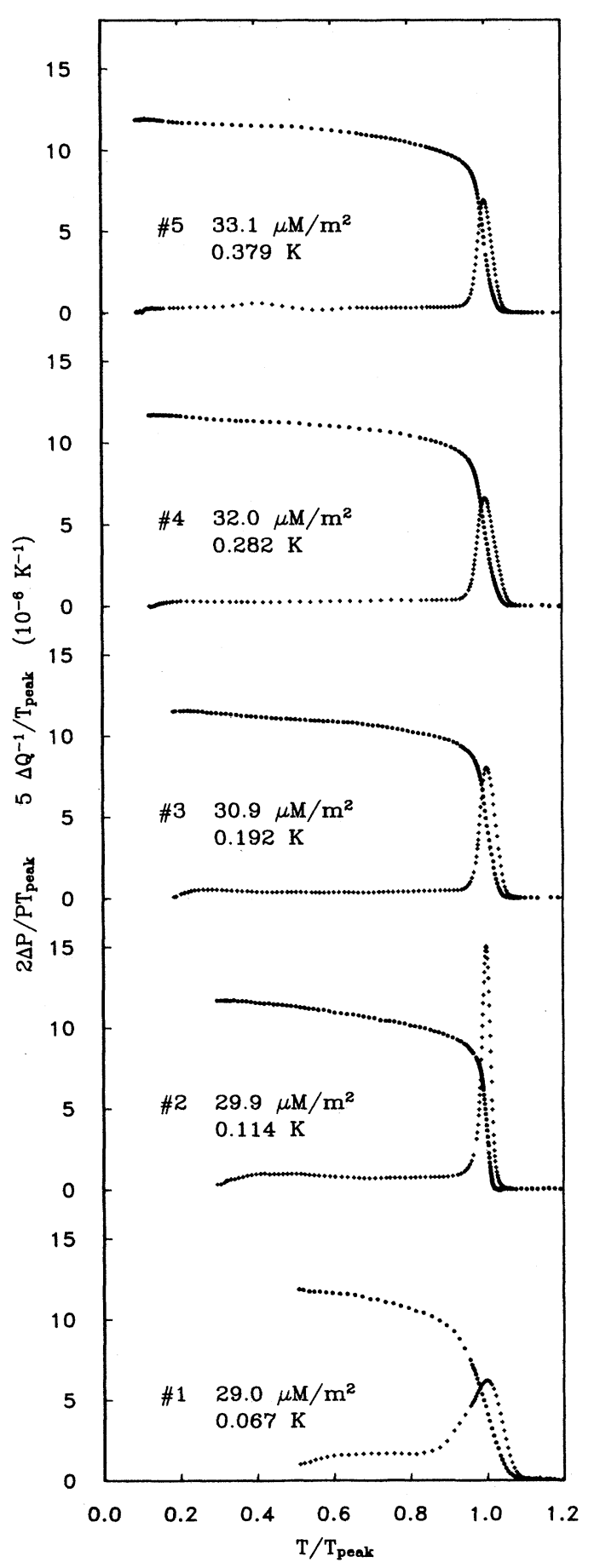

FIG. 19. Scaled period shift $2 \Delta P / P T_{\text {peak }}$ and $\Delta Q^{-1} / T_{\text {peak }}$ for different coverages. The value of $T_{\text {peak }}$ is shown below the nominal coverage for each film. The transition region, as indicated by the dissipation peak, is narrower for coverage no. 2 than for the other coverages. twice the core energy, $2 E_{C}(T)$. The relative importance of the vortex contribution to $\rho_{s}(T)$ is illustrated in Fig. 21.

The data points plotted in Fig. 21 were obtained by first averaging the scaled period curves presented in Fig. 20 and then rescaling the result with $T_{\text {peak }}=0.8 \mathrm{~K}$. This curve represents the effective superfluid density of a helium film with a zero temperature superfluid density of $43.2 \mu \mathrm{g} / \mathrm{m}^{2}$.

The dashed curve represents the temperature dependence of $\rho_{s}(T)$ assuming only vortex excitations. This curve is calculated from the full dynamic theory with $\rho_{s 0}(T)$ assumed to be temperature independent with a value given by $\rho_{s}(T=0)$. The parameter $\rho_{s 0}\left(T_{c}\right) / T_{c}$ is chosen to produce the rapid decrease of $\rho_{s}(T)$ in the correct temperature region. As one can see the resulting curve is essentially temperature independent below $T=0.5$, in agreement with our expectations for the exponential nature of the vortex pair density. From this we conclude that the low temperature scaling behavior must be a property of $\rho_{s 0}(T)$ or equivalently the nonvortex excitations.

To model this scaling behavior we have chosen a simple energy spectrum for the non-vortex excitations which correctly reproduces the temperature dependence of $\rho_{s 0}(T)$. Although a $2 \mathrm{D}$ phonon spectrum $[\epsilon(p)=c p]$ could in principle reproduce the scaling behavior, the resulting $T^{3}$ dependence of the normal density would be inconsistent with the more gradual temperature dependence of our data. To proceed beyond a simple phonon spectrum, we have taken a result from the analysis of the interacting Bose gas. Within the Bogoliubov approximation, ${ }^{41}$ the excitation spectrum is characterized by a phononlike branch with an upward dispersion at short wavelengths due to particlelike excitations, ${ }^{42}$

$$
\epsilon(p)=\left[(c p)^{2}+\left(p^{2} / 2 m\right)^{2}\right]^{1 / 2}
$$

The long-wavelength sound velocity $c$ can be expressed as the Fourier transform $\mathcal{V}(k)$ of the helium interparticle interaction potential, i.e.,

$$
c=\left(\frac{\mathcal{V}(0) n_{0}}{m}\right)^{1 / 2},
$$

where $n_{0}$ is the condensate density. ${ }^{43}$ To reproduce the observed scaling it is sufficient to define the sound velocity as

$$
c=\left[\frac{\mathcal{V}(0) \rho_{s 0}(T=0)}{m^{2}}\right]^{1 / 2},
$$

with $\mathcal{V}(0)$ assumed to be coverage independent. Given the above excitation spectrum together with our definition for the sound velocity, $\rho_{s 0}(T) / \rho_{s 0}(T=0)$ can be shown to be a function of $T / T^{*}$, where $k_{B} T^{*}$ $=\mathcal{V}(0) \rho_{s 0}(T=0) / m$. Combining this result with our initial expression, Eq. (3.1), we obtain

$$
\begin{aligned}
& \frac{2 \Delta P / P}{T_{\text {peak }}}=\mathcal{G}_{1}\left\{T / T_{\text {peak }}, l_{D}, F, \rho_{s 0}\left(T_{c}\right) / T_{c}, \mathcal{V}(0)\right\}, \\
& \frac{\Delta Q^{-1}}{T_{\text {peak }}}=\mathcal{S}_{2}\left\{T / T_{\text {peak }}, l_{D}, F, \rho_{s 0}\left(T_{c}\right) / T_{c}, \mathcal{V}(0)\right\},
\end{aligned}
$$




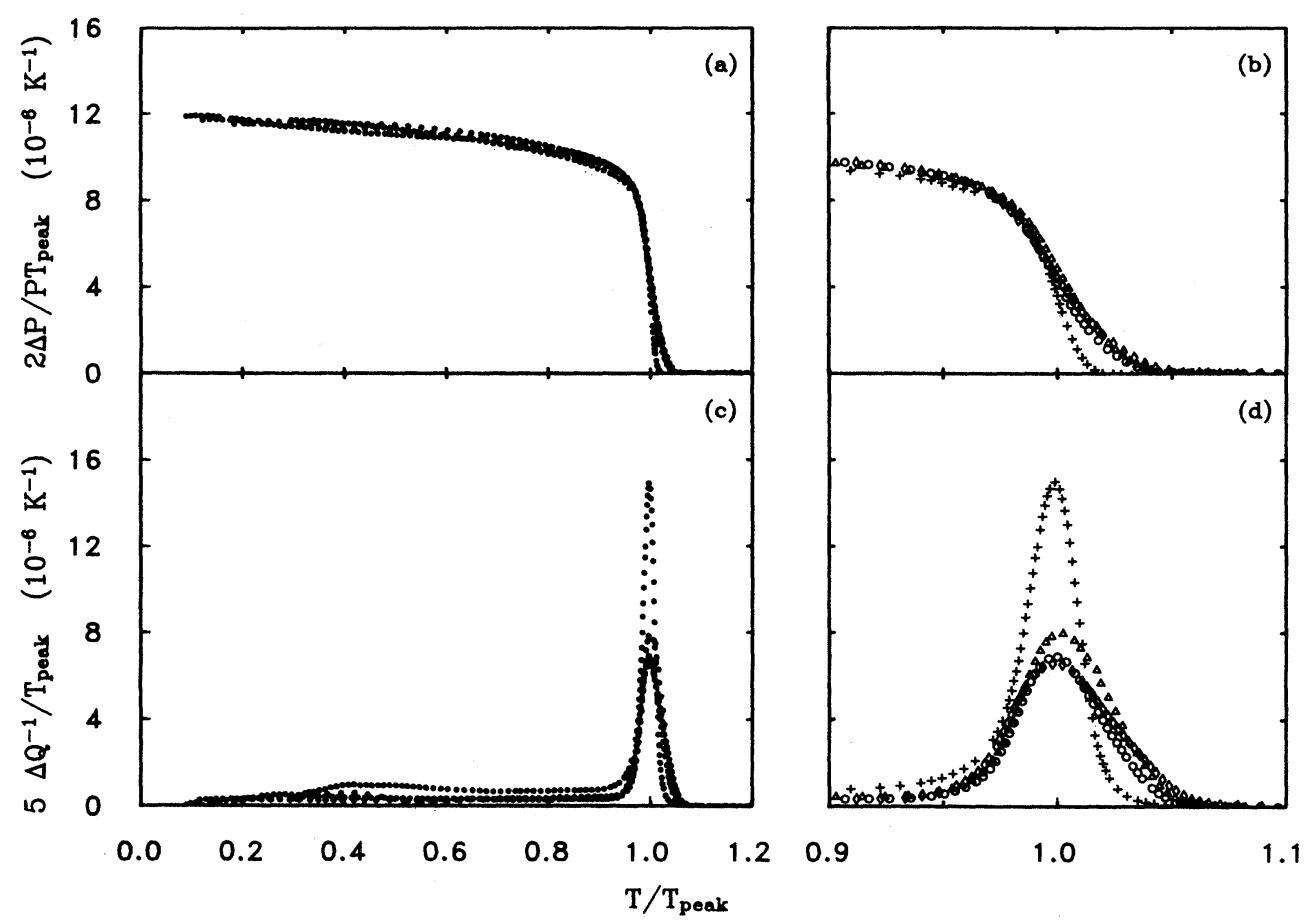

FIG. 20. Scaled data of different coverages overlapped to illustrate universal behavior. The temperature scale has been expanded in (b) and (d).

where we have again used our previous arguments to replace $T^{*}$ with $T_{\text {peak }}$. The significance of these scaling relations is that they are valid over the entire range of the data.

In fitting our data we find the inclusion of a surface ro-

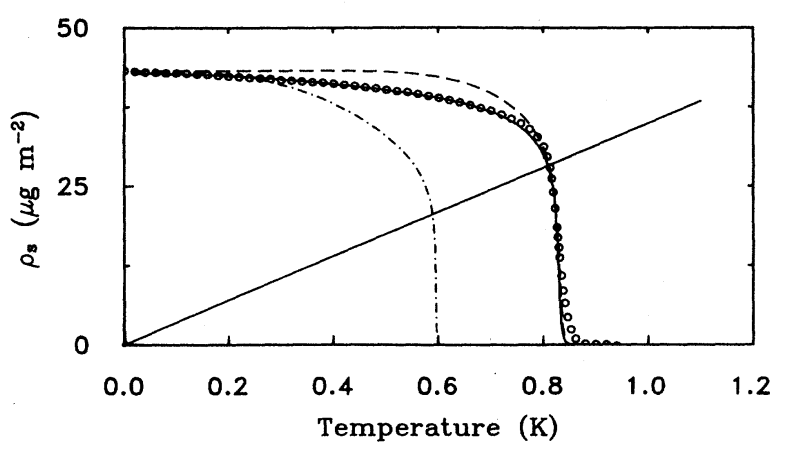

FIG. 21. Calculated superfluid density $\rho_{s}(T)$ for different normal densities. The points were obtained by first averaging the scaled period data $\left(2 \Delta P / P T_{\text {peak }}\right.$ vs $\left.T / T_{\text {peak }}\right)$ of coverages no. 2 , no. 3 , no. 4 , and no. 5 and then multiplying by $T_{\text {peak }}=0.8$ $K$ to obtain an effective superfluid density curve. The dashed line was calculated assuming only vortex excitations and is therefore only weakly temperature dependent at low temperatures. The solid curve represents the full dynamic theory in which the nonvortex excitations are described by a 2D Bogoliubov spectrum. (For comparison the dash-dot curve was calculated with the Bogoliubov spectrum replaced by a simple phonon spectrum with the same long-wavelength sound velocity.) ton branch not to be justified. We point out that both neutron scattering results ${ }^{44}$ and theoretical calculations $\mathrm{s}^{45}$ indicate $2 \mathrm{D}$ roton energy gaps which are typically greater than $5 \mathrm{~K}$. If these values are correct then the resulting roton contribution to $\rho_{s 0}(T)$ would be insignificant at the temperatures of our data. We also suggest that the roton signature observed in the third sound data of Rutledge et al. ${ }^{19}$ may in fact be due to vortex pairs since the normal density from both rotons and the vortex pairs is exponential at low temperatures $\left(\exp \left[-2 E_{c}(T) / k_{B} T\right]\right.$ and $\left.\exp \left(-\Delta / k_{B} T\right)\right)$.

In proposing the above excitation spectrum we attached no significance beyond that of a parametrization which reproduces the essential features of $\rho_{s 0}(T)$. In practice $\mathcal{V}(0)$ was independently determined by fitting the low-temperature-period data (corresponding to $\left.T / T_{\text {peak }}<0.6\right)$ for each coverage. By fitting $\rho_{s 0}(T)$ with only two parameters, $\rho_{s 0}(T=0)$ and $c^{2}$, we determine that $c^{2}$ is indeed proportional to $\rho_{s 0}(T=0)$ with $\mathcal{V}(0) / k_{B}$ $=19.4 \AA^{2}{ }^{46}$

With $\rho_{s 0}(T)$ specified we can continue with the analysis of the data in the transition region. As we pointed out before, even after scaling, coverage no. 2 still exhibits a narrower transition region and a larger dissipation peak. Because the data of coverages no. 3 , no. 4 , and no. 5 (and perhaps coverage no. 1) as a group exhibit universal behavior, one might suspect that coverage no. 2 is somehow anomalous. However, in comparing the data to the theoretical calculation, we find the exact opposite. It is the broad transitions which are not adequately described by the present theory. 
As outlined in Sec. I E the theoretical fits require only three additional parameters $\rho_{s 0}\left(T_{c}\right) / T_{c}, l_{D}$, and $F$ [ $\rho_{s 0}(T=0)$ becomes irrelevant once the data are scaled by $\left.T_{\text {peak }}\right] . \quad \rho_{s 0}\left(T_{c}\right) / T_{c}$ determines the curvature of $\rho_{s}(T)$ near $T_{c}$ and to a large extent the value of $\rho_{s}(T$ $=0) / T_{\text {peak }}$. The temperature dependence of the dissipation peak and its corresponding period shift are determined by the parameters $l_{D}$ and $F$. In particular the value of $F$ correlates strongly with the height of the dissipation peak while $l_{D}$ correlates strongly with its width. Consistent with the overall scaling of the period shift (except in the limited region of the dissipation peaks) we are able to fit all coverages with a single value of $\rho_{s}\left(T_{c}\right) / T_{c}=4.6 \pm 0.1 \times 10^{-5} \mathrm{~g} \mathrm{~m}^{-2} \mathrm{~K}^{-1}$. Corresponding to this value of $\rho_{s}\left(T_{c}\right) / T_{c}$ we determine $E_{c}\left(T_{c}\right) / k_{B} T_{c}$ $=3.2$ which is consistent with the small vortex contribution to $\rho_{n}$ at low temperatures. The ratio $(m / \hbar)^{2} E_{c}\left(T_{c}\right) / \rho_{s 0}\left(T_{c}\right)=3.8$ which is set by the Kosterlitz-Thouless recursion relations [Eq. (1.9)] is larger than the values obtained from a rigid rotating-core model $(\pi / 4)$ or an interacting excitation model $(\approx 1.2){ }^{47}$ Similar values for this ratio have been obtained for films adsorbed on packed powders. ${ }^{48}$

From this value of $\rho_{s 0}\left(T_{c}\right) / T_{c}$ and our analysis of $\rho_{s 0}(T)$ we can estimate the $T_{c}$ for each coverage. These $T_{c}$ values are listed in Table II. The relative shift between $T_{\text {peak }}$ and $T_{c}$ is only a few percent.

When analyzing the individual data for each coverage one quickly discovers that the small and broad peaks characteristic of coverages no. 3 , no. 4 , and no. 5 cannot be fitted with any choice of $l_{D}$ and $F$. In particular, the overall height of the dissipation peak relative to the period shift is too small to be explained by the dynamic theory. ${ }^{49}$ Surprisingly the seemingly anomalous data of coverage no. 2 is well fitted by the theory as illustrated in
Fig. 22. Parameters for the fit are shown in the inset.

The procedure for determining $l_{D}$ from the coverage no. 2 dissipation peak was complicated by the finite velocity effects discussed in Sec. II D. Fortunately the principal effect of the finite velocities is to broaden the dissipation peak principally on its low-temperature side. We therefore choose a value for $l_{D}$ which gives the best fit to the high-temperature side of the peak. Choosing a smaller value for $l_{D}$ would produce an overall broader peak but the broadening would be symmetric about $T_{\text {peak }}$ and therefore the resulting dissipation peak would fail to reproduce the observed sharp decrease on the hightemperature side of the peak. In a separate run we have taken data at approximately the same coverage but at a much lower substrate velocity. The lower-velocity dissipation peak is only $35 \%$ as wide as the peak of coverage no. 2 and agrees very well with the theoretical dissipation peak calculated from the parameters shown. For comparison the data taken at a smaller substrate velocity are plotted in Fig. 22 using squares. We believe that this value for $l_{D}$ represents the true low-velocity behavior of the helium film.

Our fitted value of $l_{D}=5.75$ corresponds to a dynamic length scale of $r_{D} / r_{0}=314$. The effective diffusion rate $D / r_{0}^{2}=5.6 \times 10^{7} \mathrm{sec}^{-1}$ is much smaller than values obtained for coverages with $T_{\text {peak }}>1 \mathrm{~K}$. Thermal transport measurements ${ }^{7,50,51}$ and torsional oscillator experiments ${ }^{7,31}$ give values that range from $10^{10}$ to $10^{11} \mathrm{sec}^{-1}$. Adams and Glaberson ${ }^{17}$ using a torsional oscillator technique measure the vortex diffusivity $D$ directly by injecting a known density of free vortices into the helium film by means of a rotating cryostat. They obtain $D=0.5$ to $1.5(\hbar / \mathrm{m})$ for helium films with $T_{\text {peak }}=2.0$ to $1.3 \mathrm{~K}$, respectively. If we assume the vortex core diameter to be approximately $1.5 \AA$, their effective diffusion rate be-

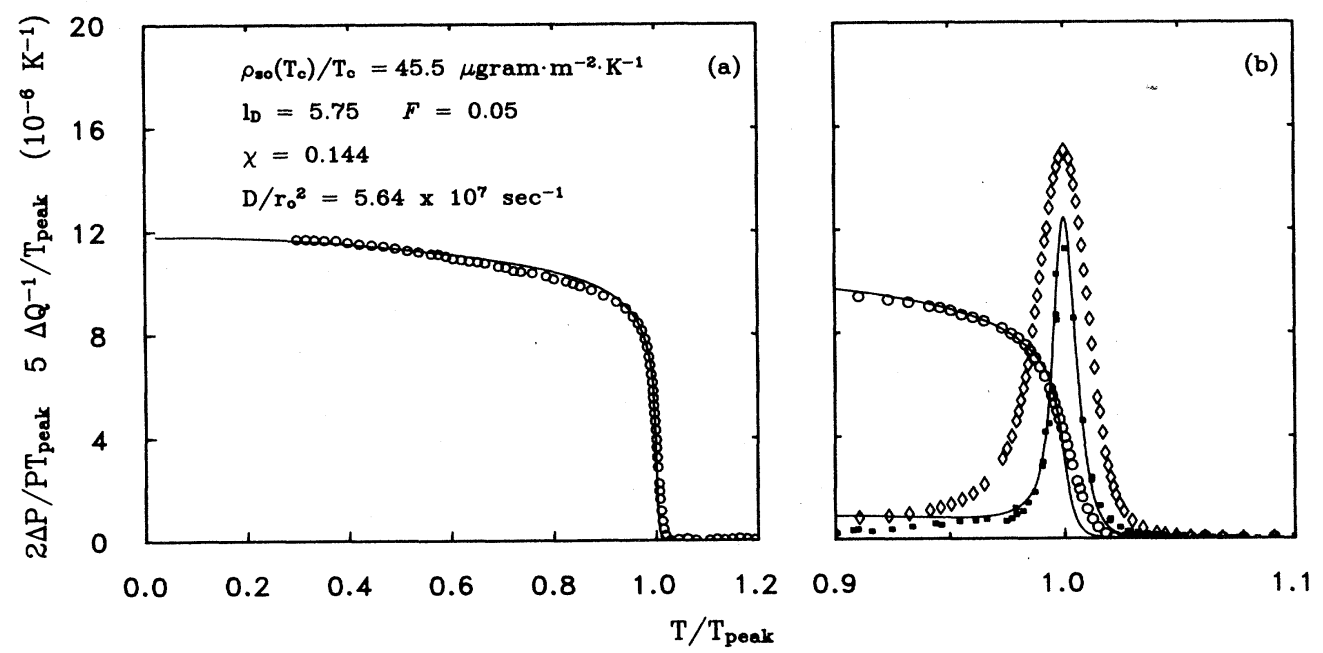

FIG. 22. The reduced period and $Q^{-1}$ data of coverage no. 2 compared to calculations based on the dynamic theory of AHNS. $\rho_{s 0}\left(T_{c}\right) / T_{c}$ was chosen to fit the average reduced period shift, while $l_{D}$ and $F$ were chosen to agree with data taken at a lower substrate velocity (indicated by the square symbols). 


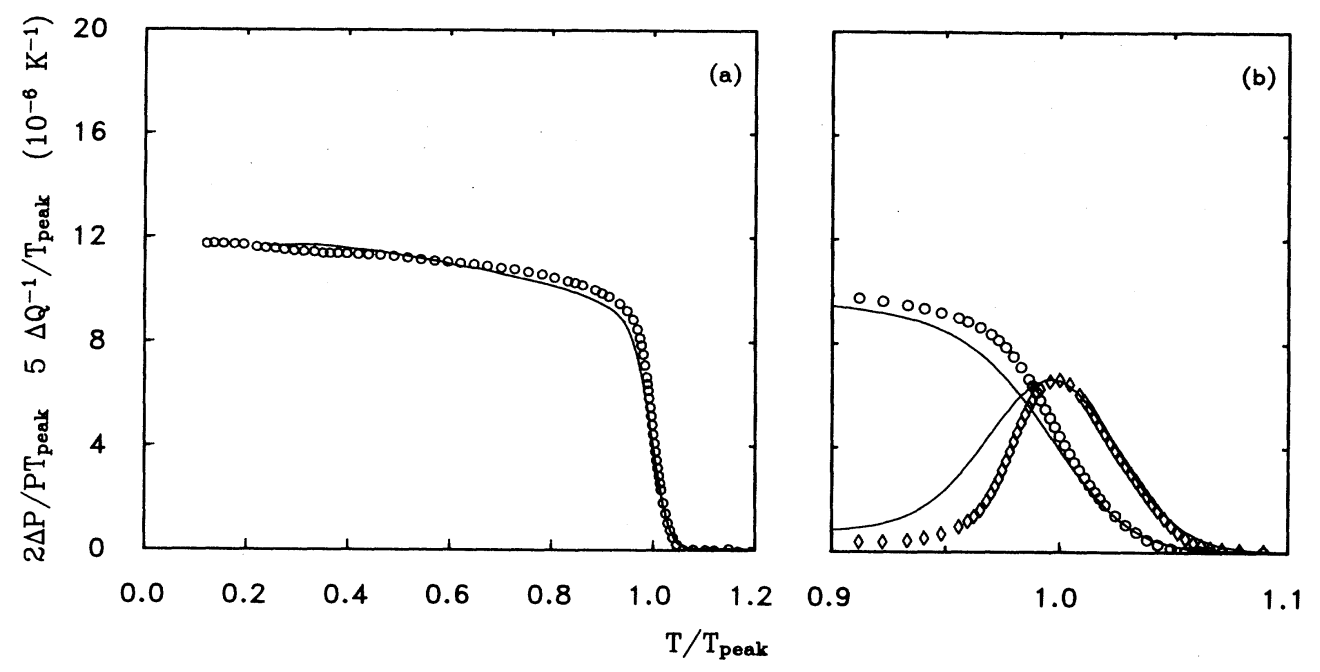

FIG. 23. Calculated superfluid response assuming spatial inhomogeneities. Only a narrow distribution of $T_{\text {peak }}$ values (corresponding to a standard deviation of only $2.5 \%$ ) is required to obtain the observed broadening.

comes $D / r_{0}^{2}=1.4$ to $4.2 \times 10^{12} \mathrm{sec}^{-1}$.

From the general scaling arguments presented, we would expect that the parameters given in Fig. 22 to apply equally well to all coverages. But, as we have previously mentioned, the data with the broader peaks cannot be explained by the present theory. A possible clue to understanding this behavior can be found in the area of each of the dissipation peaks. Although the dissipation peaks have different shapes, the area of each peak approximately scales with $T_{\text {peak }}$. This strongly suggests that in the higher coverages the transitions are broadened by an external mechanism which preserves the overall scaling behavior with coverage. One possible candidate would be macroscopic inhomogeneities of the areal density.

To simulate the effect of such inhomogeneities we have taken the scaled data corresponding to coverage no. 2 and have generated the effective superfluid response of an inhomogeneous film. Dividing our total surface area into 25 different regions, we assign each region a different $T_{\text {peak }}$ assuming a Gaussian distribution. The contribution from each region is calculated by rescaling the data of coverage no. 2 by the value of $T_{\text {peak }}$ for that region. The total dissipation is compared with the data in Fig. 23. As expected, the low-temperature response is hardly affected by this process whereas the dissipation peak is significantly broadened. Only a deviation of $2.5 \%$ in the $T_{\text {peak }}$ distribution is required to obtain the indicated broadening. Although the fit is not perfect and certainly could be improved by adjusting the $T_{\text {peak }}$ distribution, we believe that spatial inhomogeneities clearly can account for the principal features of the data. Unfortunately this does not explain why coverage no. 2 is not similarly affected by these spatial inhomogeneities.

\section{SUMMARY}

We have made measurements of the superfluid response in thin helium films adsorbed on a Mylar sub- strate. Superfluid coverages corresponding to $1.2,2.0$, $3.1,4.1$, and $5.2 \mu \mathrm{M} / \mathrm{m}^{2}$ were examined. Since the transition temperatures of these films were less than $0.5 \mathrm{~K}$, no corrections for gas desorption were necessary.

Our Mylar substrate comprises part of the moment of inertia of a high- $Q$ torsional oscillator. Consequently, changes in the superfluid mass or dissipation of the adsorbed helium film produce changes in the oscillator period and amplitude. As has been reported in other experiments, we find that a finite coverage of helium must be adsorbed before superfluidity is observed. (This initial coverage is $27.85 \mu \mathrm{M} / \mathrm{m}^{2}$ in our experiments.) We find that the temperature dependence of the period and amplitude background above the superfluid transition for each coverage is significantly different from that of the empty cell background but is independent of the coverage. This suggests that this anomalous contribution is an interfacial effect originating in the initially adsorbed helium which does not exhibit superfluidity. In determining the superfluid response, we have assumed that this contribution is unaffected by the superfluid transition and therefore should be incorporated as a part of the empty cell background.

At low temperatures the superfluid density, $\rho_{s}(T)$, for each coverage decreases slowly with increasing temperature. Eventually $\rho_{s}(T)$ decreases more rapidly at a temperature which roughly scales with the superfluid coverage in agreement with the predictions of the KosterlitzThouless theory. By including the dynamics of the vortex pairs and free vortices, AHNS are able to explain the gradual decrease of $\rho_{s}(T)$ as well as the narrow peak in the film dissipation.

Comparing the period and dissipation data for different coverages, we observe a definite scaling of the data with coverage. By rescaling the data by $T_{\text {peak }}$, the temperature of the dissipation peak, we find that to a large extent both the period and dissipation data are universal functions of $T / T_{\text {peak }}$, independent of the coverage. Univer- 
ity in the region of the superfluid transition was expected, as it is a direct consequence of the vortex nature of the transition. However, the universality observed for temperatures well below the transition region was unexpected and indicates that the non-vortex excitations also obey some form of scaling relation.

Using as a guide the results from the analysis of an interacting Bose gas we have developed a phenomenological model for the energy spectrum of these nonvortex excitations. The energy spectrum is linear in the momentum at long wavelengths but has an upward dispersion characteristic of a free particle at short wavelengths. To reproduce the scaling of the low-temperature period data with coverage, it is sufficient to choose the longwavelength sound velocity to be proportional to the square root of the superfluid areal density at $T=0$. Given this ansatz, the low-temperature data from different coverages can be fitted with a single parameter.

By incorporating our analysis of the nonvortex excitations with the dynamic theory of AHNS, we obtain a complete description of the superfluid response over the entire temperature region of interest. The calculated scaled response only depends on three new parameters: $\rho_{s 0}\left(T_{c}\right) / T_{c}$, the effective interaction strength between vortices at $T_{c} ; F$, the proportionality between the hightemperature correlation length and the free vortex density; and $l_{D}$, the dynamical length scale set by the vortex diffusivity.

From our analysis we find that each coverage is well described by a single value of $\rho_{s 0}\left(T_{c}\right) / T_{c}=45.5 \mu \mathrm{g} \mathrm{m}^{-2}$ $\mathrm{K}^{-1}$. Since our measured dissipation peaks are much broader than those measured in thicker films $\left(T_{\text {peak }}>1\right.$ $\mathrm{K})$, the fits to the data require a much smaller value for $l_{D}$ than has been previously reported. In terms of a diffusion rate we require $D / r_{0}^{2}=5.6 \times 10^{7} \mathrm{sec}^{-1}$, where $r_{0}$ is the vortex core radius [taking $r_{0} \approx 1.5 \AA$, we obtain a vortex diffusivity of only $2 \times 10^{-5}(\hbar / \mathrm{m})$ ].

These values represent a dramatic reduction from the values found in thicker films $\left(\approx 10^{11} \mathrm{sec}^{-1}\right)$. This reduction could be ascribed to either a change in the vortex diffusivity or a change in the vortex core radius. For example, if at low coverages, vortex pinning by substrate imperfections were dramatically enhanced, the overall vortex diffusivity would decrease. Similarly an increase in the vortex core radius could produce the decrease in the diffusion rate. In analyzing third sound measurements on thin helium films adsorbed on porous media, Kotsubo and Williams ${ }^{48}$ find core radii as large as $50 \AA$. Haldane and $\mathrm{Wu}^{52}$ point out that due to the atomic nature of helium films the vortex core cannot be localized within an area smaller than the mean area per particle. ${ }^{53}$ Thus one should expect the core radius to roughly scale with $\left[\rho_{s 0}(T=0)\right]^{-1 / 2}$. If we take the diffusivity $D$ to be on the order of $(\hbar / m)$, then our measured diffusion rate would imply a core radius of $r_{0} \approx 168 \AA$ which is much larger than the interparticle spacing estimated from the superfluid coverage $(\approx 9.8 \AA)$. Our oscillator technique unfortunately cannot distinguish between these two different interpretations. Clearly a direct measurement of $D$ of the type made by Adams and Glaberson ${ }^{17}$ would be valuable in understanding these anomalously small values of $D / r_{0}^{2}$.

In addition to the very small vortex diffusion rates we observe additional broadening for certain coverages. We have interpreted this departure from the scaling behavior to indicate the presence of macroscopic spatial inhomogeneities in the film. By assuming a narrow distribution of areal densities of only $2.5 \%$ we can produce an additional broadening comparable to the observed values. The coverage dependence of this additional broadening, however, is still a mystery. Although macroscopic imperfections or energetic inhomogeneities could easily produce such distributions in the areal density, it is difficult to understand in our experiment why a thinner film should be less affected. In fact, more recent data ${ }^{54}$ indicate that the additional broadening in the dissipation peaks extends over a much wider range of coverages and appears to vary periodically with coverage.

From the data it is not possible to determine whether this additional broadening is intrinsic to the helium film or whether it is induced by the substrate. Extending these studies to other substrates may help to decide this question. In particular, these measurements need to be made on well characterized substrates (such as graphite) so that the coverage dependence can be correlated with known properties of the surface. Preplating with other gases such as krypton to reduce surface inhomogeneities would also be informative. These studies would also test whether the scaling behavior of the nonvortex excitations is universal.

In conclusion, we have presented data on the superfluid response of thin helium films. We have shown that our data can be described over the entire range of temperature by a scaling function that is independent of the coverage. From this analysis we conclude that the broad transitions observed at certain coverages is caused by an external mechanism which preserves the overall scaling with coverage.

\section{ACKNOWLEDGMENTS}

The experimental portion of the work reported here was carried out at Cornell University in the Laboratory of Atomic and Solid State Physics with support from the National Science Foundation under Grant No. NSFDMR-8418605 and with support from the Cornell Materials Science Center through Grant No. NSF-DMR82-17227A, MSC Report 6537. J.D.R. would like to thank the Aspen Center for Physics for hospitality during the period of this research. The authors are also grateful to M. C. Cross, P. C. Hohenberg, P. M. L. Minnhagen, and D. D. Osheroff for stimulating discussions concerning the interpretation of these results. One of us (G.A.) would like to thank AT\&T Bell Laboratories where some of this analysis was done. In addition he would like to thank G. Verdi for providing sufficient acoustic diversion during the composing phase of this work. We acknowledge the support of the Robert A. Welch Foundation (Grant No. A-1065) and the National Science Foundation (Grant No. DMR-8415893). 


\section{APPENDIX: VAPOR-PRESSURE CALIBRATION}

In general the total film coverage (the number of helium atoms adsorbed on the substrate) is not a fixed quantity but can vary with temperature because of desorption into the gas. At low coverages and temperatures (both $T$ and $T_{c}$ below $1 \mathrm{~K}$ ) these variations of the film coverage can be safely ignored and the coverage can be determined solely from the total amount of helium admitted to the cell. At higher temperatures, corrections must be made to the coverage. This is particularly true when the open volume of the experimental cell is large compared to the surface area of the system.

To model the temperature dependence of the pressure of a relatively thick film, we shall assume the gas pressure in equilibrium with the film to be given by,

$$
P(T)=P_{0}(T) \exp \left[V(d) / k_{B} T\right],
$$

where $P$ is the pressure of the gas at temperature $T$ and $P_{0}(T)$ is the saturated vapor pressure at that temperature. $V(d)$ is the attractive substrate potential evaluated at the surface of the film with thickness $d$. For $V(d)$ we shall use the van der Waals potential which for small distances from the substrate falls off as $d^{-3}$; i.e., $V(d)=-\alpha k_{B} / d^{3}$.

The total helium $N_{T}$ in the cell is either in the vapor or in the adsorbed film,

$$
N_{T}=N_{\text {film }}+N_{\text {vapor }},
$$

where $N_{\text {vapor }}$ is given by the pressure and the ideal gas law; i.e., $N_{\text {vapor }}=P V / R T$. Since the attractive potential exerts on the film an effective pressure which increases with decreasing distance from the substrate, the density of the film will vary with the distance from the substrate. We shall approximate this change in density by dividing the total thickness $d$ into an initial layer of thickness $d_{s}$ with an assigned density equal to that of the solid $(0.191$ $\mathrm{g} / \mathrm{cm}^{3}$ ) and an outer layer of thickness $d_{1}$ with an assigned density equal to that of the liquid $\left(0.145 \mathrm{~g} / \mathrm{cm}^{3}\right)$. We arbitrarily equate the high-density layer to the nonsuperfluid layer found by extrapolating the $T_{\text {peak }}$ versus coverage curve to $T=0$. Substituting the value for the inert layer obtained in Sec. III A we find that $\rho_{\text {solid }}=27.85 \mu \mathrm{M} / \mathrm{m}^{2}$.

Our expression for $N_{T}$ becomes

$$
N_{T}=S \rho_{\mathrm{solid}} d_{s}+S \rho_{\mathrm{liq}} d_{1}+P V / R T
$$

or in terms of the total thickness $d=d_{1}+d_{s}$,

$$
N_{T}=S\left(\rho_{\text {solid }}-\rho_{\text {liq }}\right) d_{s}+S \rho_{\mathrm{liq}} d+P V / R T
$$

where $S$ is the surface area and $V$ is the volume of the cell.

Substituting for $d$ from the expression for the pressure (A1) we obtain

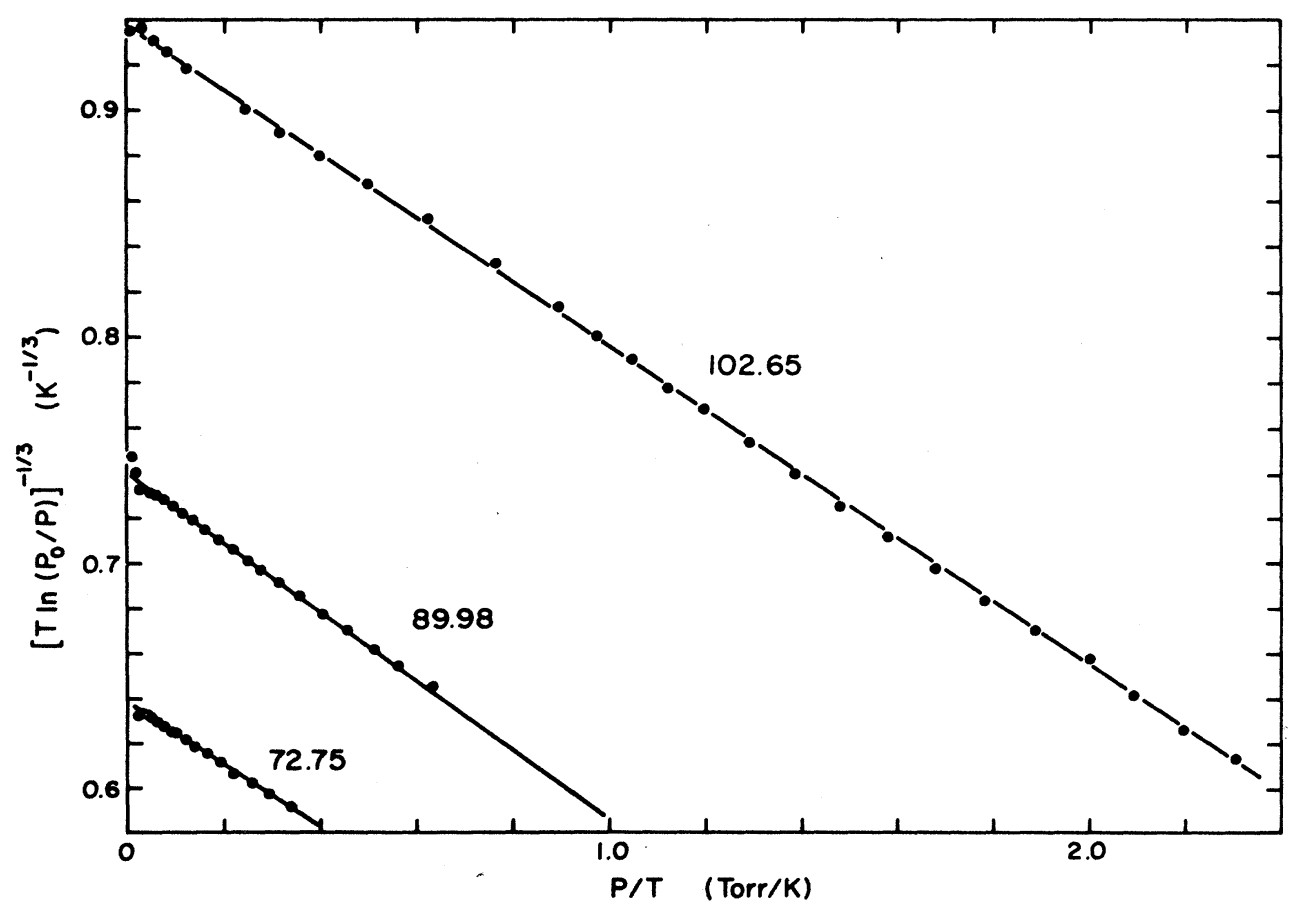

FIG. 24. Analysis of the gas pressure in equilibrium with the helium film. The total helium within the cell is indicated for each data set. From the straight-line fits we determine the van der Waal's constant of the substrate and the internal volume of the cell. 


$$
\begin{aligned}
& {\left[T \ln \left(P_{0} / P\right)\right]^{-1 / 3} }=\left(\alpha^{1 / 3} S \rho_{\text {liq }}\right) N_{T}^{\prime} \\
&+\left(\frac{V}{R \alpha^{1 / 3} S \rho_{\text {liq }}}\right)(P / T) \\
& \text { where } N_{T}^{\prime}=N_{T}-S\left(\rho_{\text {solid }}-\rho_{\text {liq }}\right) d_{s} .
\end{aligned}
$$

By plotting $\left[T \ln \left(P_{0} / P\right)\right]^{-1 / 3}$ against $(P / T)$ we determine both the van der Waals constant, $\alpha$, and the volume of the system $V$. In Fig. 24, we show this analysis for three different values of $N_{T}$. All three curves give consistent values for the parameters: $\alpha=52.05 \mathrm{~K} / L^{3}$ (where $L$ denotes layer) and $V=0.843 \mathrm{~cm}^{3}$. The value of $\alpha$ is somewhat larger than one would expect in comparison with other dielectric materials but does fall within the range quoted by Bienfait et al. ${ }^{55}$ The volume is consistent with the measured volume of the cell ( $V_{\text {cell }}=0.613$ $\left.\mathrm{cm}^{3}\right)$ and pressure gauge $\left(V_{\text {gauge }}=0.10 \mathrm{~cm}^{3}\right)$ and estimates of the capillary volume.
*Present address: IBM Thomas J. Watson Research Center, Yorktown Heights, NY 10598.

${ }^{1}$ J. M. Kosterlitz and D. J. Thouless, J. Phys. C 6, 1181 (1973); J. M. Kosterlitz, ibid. 7, 1046 (1974).

${ }^{2}$ V. Ambegaokar, B. I. Halperin, D. R. Nelson, and E. Siggia, Phys. Rev. B 21, 1806 (1980).

${ }^{3}$ B. A. Huberman, R. J. Myerson, and S. Doniach, Phys. Rev. Lett. 40, 780 (1978); R. J. Myerson, Phys. Rev. B 16, 3203 (1977).

${ }^{4}$ D. J. Bishop and J. D. Reppy, Phys. Rev. Lett. 40, 1727 (1978).

${ }^{5}$ M. Chester and L. C. Yang, Phys. Rev. Lett. 31, 1377 (1973).

${ }^{6}$ I. Rudnick, Phys. Rev. Lett. 40, 1454 (1978).

${ }^{7}$ G. Agnolet, S. L. Teitel, and J. D. Reppy, Phys. Rev. Lett. 47, 1537 (1981).

${ }^{8}$ J. Maps and R. B. Hallock, Phys. Rev. Lett. 47, 1533 (1981).

${ }^{9}$ A. P. Young, in Ordering in Strongly Fluctuating Condensed Matter Systems, edited by T. Riste (Plenum, New York, 1979), p. 271.

10J. M. Kosterlitz and D. J. Thouless, in Progress in Low Temperature Physics, edited by D. F. Brewer (North-Holland, Amsterdam, 1978), Vol. 7, p. 1; D. R. Nelson, in Phase Transitions and Critical Phenomena, edited by C. Domb and J. L. Lebowitz (Academic, London, 1983), Vol. 7, p. 1; B. I. Halperin, in Physics of Low Dimensional Systems, edited by Y. Nagaoka and S. Hikami (Publication Office, Progress of Theoretical Physics, Kyoto, 1979), p. 54.

${ }^{11}$ W. F. Saam, Phys. Rev. B 23, 1485 (1981).

${ }^{12}$ D. R. Nelson and J. M. Kosterlitz, Phys. Rev. Lett. 39, 1201 (1977).

${ }^{13}$ W. F. Vinen, in Progress in Low Temperature Physics, edited by C. J. Gorter (North-Holland, Amsterdam, 1961), Vol. 3, p. 1 , and references therein.

${ }^{14}$ V. Ambegaokar and S. Teitel, Phys. Rev. B 19, 1667 (1979).

${ }^{15}$ This is analogous to the result obtained using a GinsburgLandau formalism for superconductors in which $E_{c}$ is taken to be proportional to $\rho_{s 0}$.

16P. Minnhagen and M. Nylen, Phys. Rev. B 31, 1693 (1985).

${ }^{17}$ M. Kim and W. I. Glaberson, Phys. Rev. Lett. 52, 53 (1984); P. W. Adams and W. I. Glaberson, Phys. Rev. B 35, 4633 (1987); although they demonstrate that the vortex diffusivity $D$ does have a temperature dependence, the influence of the diffusivity on the reduced period and dissipation is principally in the region of $T_{c}$. We therefore can approximate the diffusion constant by its average value at $T_{c}$.

${ }^{18}$ W. F. Saam and M. W. Cole, Phys. Rev. B 11, 1086 (1975).

${ }^{19}$ J. E. Rutledge, W. L. McMillan, J. M. Moche1, and T. E. Washburn, Phys. Rev. B 18, 2155 (1978).

${ }^{20}$ Instead of fixing the values of $\operatorname{Re}[\epsilon(\omega)]$ and $\operatorname{Im}[\epsilon(\omega)]$ at $T_{+}$ one could continue to evaluate the pair contributions to $\operatorname{Re}[\epsilon(\omega)]$ and $\operatorname{Im}[\epsilon(\omega)]$ beyond $T_{+}$. Because the free vortices dominate the total response at this temperature both calculations give equivalent values for the reduced period and dissipation.

${ }^{21}$ E. L. Andronikashvili, Zh. Eksp. Teor. Fiz. 16, 780 (1946).

${ }^{22}$ Alloy Beryco 25 , heat treated at $316^{\circ} \mathrm{C}$ for $3 \mathrm{~h}$ to achieve maximum hardness; Kawecki Berylco Industries Inc., NY.

${ }^{23}$ DuPont Company, Wilmington, DE.

${ }^{24}$ Emerson Cummings Inc., Canton, MA.

${ }^{25}$ This proved to be a mistake since $\mathbf{N}$ grease will fracture upon cooling. Epoxy or silicone grease would have been better choices.

${ }^{26}$ EG\&G Instruments, Princeton, NJ.

${ }^{27}$ D. S. Greywall and P. A. Busch, Rev. Sci. Instrum. 51, 509 (1980).

${ }^{28} \mathrm{~K}$. W. Takanis, N. W. Pennings, P. Das, and R. deBruyn Ouboter, Physica 56, 168 (1971), constructed by Dr. E. N. Smith.

${ }^{29}$ D. S. Greywall and P. A. Busch, J. Low Temp. Phys. 46, 451 (1982).

${ }^{30}$ D. F. McQueeney, Ph.D. thesis, Cornell University (unpublished).

${ }^{31}$ D. J. Bishop, Ph.D. thesis, Cornell University, 1978 (unpublished); D. J. Bishop and J. D. Reppy, Phys. Rev. B 22, 5171 (1980); D. J. Bishop, J. E. Berthold, J. M. Parpia, and J. D. Reppy, ibid. 24, 5047 (1981).

${ }^{32}$ Yasu Takano (private communication).

${ }^{33}$ This phenomena has also been seen in other nonmetallic systems; R. N. Kleiman, G. Agnolet, and J. D. Bishop, Phys. Rev. Lett. 59, 2079 (1987).

${ }^{34}$ B. C. Crooker, Ph.D. thesis, Cornell University (unpublished).

${ }^{35}$ M. A. Paalanen, D. J. Bishop, and H. W. Dail, Phys. Rev. Lett. 46, 664 (1981).

${ }^{36}$ A. Leggett, Physica Fennica 8, 125 (1973).

${ }^{37}$ D. J. Bishop and J. D. Reppy, Phys. Rev. B 22, 5171 (1980).

${ }^{38}$ K. A. Gillis, S. Volz, and J. M. Mochel, J. Low Temp. Phys. 60, 173 (1984).

${ }^{39}$ In some ways this is reminiscent of the behavior observed in bulk helium in which the vorticity is found to be reproducible after thermal cycling above and below $T_{c}$. D. D. Awschalom and K. W. Schwarz, Phys. Rev. Lett. 52, 136 (1984).

${ }^{40}$ D. A. Browne and S. Doniach, Phys. Rev. B 25, 136 (1982).

${ }^{41}$ F. N. Bogoliubov, J. Phys. (Moscow) 11, 23 (1947).

${ }^{42}$ This spectrum is very similar to that derived by Rutledge et al. (Ref. 19) from a quantum hydrodynamic approach.

${ }^{43} \mathrm{~A}$ general treatment of the 3D problem can be found in A. L. Fetter and J. D. Walecka, Quantum Theory of Many-Particle 
Systems (McGraw-Hill, New York, 1971), pp. 215-223.

${ }^{44}$ W. Thomlinson, J. A. Tarven, and L. Passell, Phys. Rev. Lett. 44, 266 (1980); H. J. Lauter, H. Wiechert, and C. Tiky, in Proceedings of the 16th International Conference on Low Temperature Physics, edited by P. F. De Chatel, M. Durieux, and P. F. A. Klinkenberg [Physica 107B + C, 239 (1981)].

${ }^{45}$ T. C. Padmore, Phys. Rev. Lett. 32, 826 (1974).

${ }^{46} \mathrm{It}$ is interesting to note that fitted value for $\mathcal{V}(0) / k_{B}$ is very close numerically to $(m / 2)\left[T_{c} / \rho_{s}\left(T_{c}^{--}\right)\right]$or equivalently $(\pi / 4)\left[(\hbar / m)^{2} / m k_{B}\right]$.

${ }^{47}$ R. J. Donnelly and P. H. Roberts, Proc. R. Soc. London, Ser. A 321, 519 (1969).

${ }^{48}$ V. Kotsubo and G. A. Williams, Phys. Rev. B 33, 6106 (1986).

${ }^{49}$ Although the heights of the dissipation peaks can be reproduced by setting $F$ to zero (i.e., no free vortices) the overall theoretical fit is very poor.
${ }^{50}$ J. Maps and R. B. Hallock, Phys. Rev. B 27, 5491 (1983).

${ }^{51}$ D. Finotello and F. M. Gasparini, Phys. Rev. Lett. 55, 2156 (1985).

${ }^{52}$ F. D. M. Haldane and Y. Wu, Phys. Rev. Lett. 55, 505 (1982).

${ }^{53}$ G. A. Williams, in Proceedings of the 18 th International Conference on Low Temperature Physics, edited by Y. Nagaoka [Jpn. J. Appl. Phys. 26, 505 (1987)]. This idea is applied to the analysis of the superfluid transition in packed powders and porous media.

${ }^{54}$ D. F. McQueeney, G. Agnolet, G. K. S. Wong, and J. D. Reppy, in Proceedings of the 18th International Conference on Low Temperature Physics, edited by Y. Nagaoka [Jpn. J. Appl. Phys. 26, 79 (1987)].

${ }^{55}$ M. Bienfait, J. G. Dash, and J. Stoltenberg, Phys. Rev. B 21, 2765 (1980). 